\title{
Endothelial transplantation rejuvenates aged hematopoietic stem cell function
}

\author{
Michael G. Poulos, ${ }^{1,2,3}$ Pradeep Ramalingam, ${ }^{1,2,3}$ Michael C. Gutkin, ${ }^{1,2,3}$ Pierre Llanos, ${ }^{4}$ Katherine Gilleran, ${ }^{4}$ \\ Sina Y. Rabbany, ${ }^{4}$ and Jason M. Butler ${ }^{1,2,3}$ \\ DDepartment of Medicine, ${ }^{2}$ Department of Surgery, and ${ }^{3}$ Ansary Stem Cell Institute, Weill Cornell Medical College, New York, New York, USA. ${ }^{4}$ Bioengineering Program, \\ DeMatteis School of Engineering and Applied Science, Hofstra University, Hempstead, New York, USA.
}

\begin{abstract}
Age-related changes in the hematopoietic compartment are primarily attributed to cell-intrinsic alterations in hematopoietic stem cells (HSCs); however, the contribution of the aged microenvironment has not been adequately evaluated. Understanding the role of the bone marrow (BM) microenvironment in supporting HSC function may prove to be beneficial in treating age-related functional hematopoietic decline. Here, we determined that aging of endothelial cells (ECs), a critical component of the BM microenvironment, was sufficient to drive hematopoietic aging phenotypes in young HSCs. We used an ex vivo hematopoietic stem and progenitor cell/EC (HSPC/EC) coculture system as well as in vivo EC infusions following myelosuppressive injury in mice to demonstrate that aged ECs impair the repopulating activity of young HSCs and impart a myeloid bias. Conversely, young ECs restored the repopulating capacity of aged HSCs but were unable to reverse the intrinsic myeloid bias. Infusion of young, HSC-supportive BM ECs enhanced hematopoietic recovery following myelosuppressive injury and restored endogenous HSC function in aged mice. Coinfusion of young ECs augmented aged HSC engraftment and enhanced overall survival in lethally irradiated mice by mitigating damage to the BM vascular microenvironment. These data lay the groundwork for the exploration of EC therapies that can serve as adjuvant modalities to enhance HSC engraftment and accelerate hematopoietic recovery in the elderly population following myelosuppressive regimens.
\end{abstract}

\section{Introduction}

The number of elderly individuals is expected to double in the next 40 years, creating an increased burden of treatment for aging-related disorders, including cardiovascular disease and cancer (1-4). Aging of the hematopoietic system is associated with a decline in adaptive immunity (5), an increased incidence of anemia (6), and a predisposition to myeloid neoplasms $(7,8)$. Hematopoietic stem cells (HSCs) show an increase in immunophenotypically defined cells with age, a decrease in their long-term reconstitution abilities, and a significant increase in myeloid-biased cell output at the expense of lymphopoiesis (9-15). These studies clearly describe the cell-intrinsic HSC alterations that lead to aging-related hematopoietic deficiencies. While the cell-autonomous changes in the HSC that promote aging-related changes in hematopoiesis are more well defined, the contribution of the aged bone marrow (BM) microenvironment in promoting aged hematopoietic phenotypes is poorly understood $(16,17)$.

The adult BM microenvironment is a highly specialized cellular niche composed of vascular endothelial cells (ECs) and perivascular stromal constituents that support HSC maintenance and hematopoietic homeostasis (18). Within the BM hematopoietic microenvironment, the vascular endothelium is indispensable

Related Commentary: p. 3921

Conflict of interest: The authors have declared that no conflict of interest exists.

Submitted: March 10, 2017; Accepted: September 5, 2017.

Reference information: / Clin Invest. 2017;127(11):4163-4178.

https://doi.org/10.1172/JC193940. for supporting HSC quiescence, self-renewal, and differentiation into lineage-committed progeny (19-25). The aged BM microenvironment has also been shown to influence hematopoietic aging in young HSCs (26-30). While ECs are a critical component of the HSC niche $(19,20,23)$, the individual role of aged ECs in the process of hematopoietic aging has not been examined (27). Here, we explore the idea that aged ECs are sufficient to promote aging of young HSCs and that the infusion of young ECs can be exploited to improve age-related hematopoietic deficiencies.

In this study, we used cultured BM-derived endothelium from young and aged mice to evaluate whether an age-dependent dysregulation of the $\mathrm{BM}$ endothelial niche is sufficient to disrupt the homeostatic HSC-supportive microenvironment and drive aging-associated hematopoietic phenotypes. Using an established ex vivo coculture system, we demonstrated that culturing of young hematopoietic stem and progenitor cells (HSPCs) on aged endothelium inhibited long-term HSC repopulating activity in a competitive transplantation setting and promoted a myeloid bias at the expense of B and T cell lymphopoiesis. Moreover, aged HSPCs cultured on young endothelium showed a marked increase in hematopoietic reconstitution. These results extended to endothelial infusions in young and aged mice, in which aged BM-derived ECs failed to support endogenous hematopoietic recovery following myelosuppressive irradiation and imparted a myeloid bias in young mice; conversely, infusions of young ECs enhanced HSC activity and increased $\mathrm{B}$ and $\mathrm{T}$ cell output in young and aged animals. Moreover, young EC coinfusions enhanced aged HSC transplantation (HSCT) and overall survival in a limiting dilution setting through 
protection of the endogenous BM vascular niche. These data lay the groundwork for the development of cellular therapies that can serve as adjuvant modalities to enhance hematopoietic recovery in the elderly population following myelosuppressive treatments to ultimately protect patients from severe morbidities and mortality associated with the treatment of hematological disorders.

\section{Results}

Characterization of the aged BM microvasculature. To examine potential aging-related alterations in $\mathrm{BM}$ endothelium, the vasculature of young ( 3 months old) and aged ( 24 months old) C57B/6 mice was intravitally labeled with an EC-specific antibody raised against VECAD to assess endothelial morphology in the BM microenvironment. Aged mice displayed distinct changes in vascular morphology, which appeared dilated and more discontinuous and suggested a potential change in vascular integrity (Figure 1A). We next examined vascular permeability using i.v. injection of Evans blue dye. Femurs from aged mice had a significant increase in vascular leakiness, as measured by dye extravasation (Figure 1, B and C). We observed that the frequency of $\mathrm{VECAD}^{+} \mathrm{CD} 31^{+} \mathrm{CD} 45^{-} \mathrm{TER}^{-119^{-}} \mathrm{BM}$ ECs was also significantly reduced, with a marked increase in $\mathrm{VECAD}^{-} \mathrm{CD} 31^{-}$ CD45 TER119- pan-BM stromal cells (Figure 1, D and E). BM ECs from aged mice also had metabolic dysregulation, including a significant increase in ROS (Figure 1F) and hypoxic status (Figure 1, G and $\mathrm{H})$. These observations are consistent with aging-related vascular dysfunction (31-36) and suggest that instructive endothelial niche function might also be compromised during aging.

Cultured ECs from aged mice display aging-related functional alterations. To examine changes in the hematopoiesis-instructive function of aged endothelium, we isolated BM-derived ECs from young ( 3 months old) and aged (24 months old) C57BL/6 mice, as previously described (37). Cultured ECs showed uniform cellsurface expression of VECAD by immunofluorescence (Figure 2A) and expressed pan-endothelial markers $\left(\mathrm{VECAD}^{+}\right.$and $\left.\mathrm{CD} 31^{+}\right)$, as assessed by flow cytometry (Figure 2B). We next examined agingrelated characteristics in young and aged BM ECs. While young and aged ECs did not have any differences in overall cell dimensions, aged ECs showed an increase in median cellular stiffness, as measured by atomic force microscopy (AFM) (Figure 2, C-E). An increase in vascular stiffness in vivo has been reported to be associated with aging-related senescence and a decrease in endothelial angiogenic potential (38-40). However, aged EC cultures displayed no overt senescence-related morphology or significant changes in senescence-associated $\beta$-gal (SA $\beta$-gal) activity (Figure $2, \mathrm{~A}$ and $\mathrm{H}$ ). Aged ECs had a delay in cell-cycle progression 6 hours after cellcycle synchronization that was resolved by 24 hours (Figure 2, F and $\mathrm{G})$. We next examined the angiogenic potential of aged ECs in an in vitro wound-healing assay. Aged ECs displayed a significant delay in wound healing, suggesting an age-related impairment in cell migration (Figure 2, I and J). Taken together, cultured BM-derived ECs isolated from aged mice show functional alterations in vitro that are consistent with aging-related phenotypes.

The BM vascular niche supports young HSC homeostasis in vivo through the expression of prohematopoietic factors such as Kitl, Cxcl12, and Jag1. Genetic deletion of these factors using EC-specific Tie2-Cre and Cdh5-Cre results in loss of HSC frequency and repopulating activity $(19,20,22,23)$. Secreted and cellular levels of KITL were reported to be significantly decreased within the BM of aged mice (27). In humans, plasma levels of SDF1 (CXCL12) are also diminished with aging, positively correlating with a phenotypic expansion of BM HSCs and inversely correlating with BM fat accumulation, two hallmarks of BM aging (41). To determine whether aged ECs have an aberrant expression of these critical pro-HSC factors, we performed gene expression analysis by reverse transcriptase quantitative PCR (RT-qPCR) on cultured aged ECs, which demonstrated a decrease in Kitl, Cxcl12, and NOTCH ligand expression (Figure 2K), suggesting an impaired instructive function in aged ECs.

Aged ECs promote hematopoietic aging phenotypes on cocultured young HSPCs. Aging of the hematopoietic system is, in part, functionally defined by a decrease in HSC-repopulating activity and an increase in the frequency of myeloid cell output at the expense of lymphoid cells $(8,42)$. To examine the possibility that aged ECs play a direct role in promoting aging-related hematopoietic phenotypes, we used our recently described ex vivo HSPC/EC coculture system (37). This model has been used to faithfully recapitulate in vivo endothelial NOTCH- and $\mathrm{NF}-\mathrm{\kappa B}^{-}$-mediated regulation of HSCs in the vascular niche $(23,43)$. By culturing isolated HSPCs directly on ECs in serum-free conditions, we could segregate and examine the instructive role played by aged ECs in maintaining HSPC homeostasis. To this end, CD 45.2 ${ }^{+}$whole BM (WBM) was isolated from young ( 3 months old) and aged (24 months old) mice and depleted of lineage-committed hematopoietic cells.

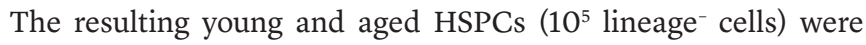
plated on young ( 3 months old) and aged (24 months old) ECs for 14 days in serum-free media with supplementation of $50 \mathrm{ng} / \mathrm{ml}$ soluble KITL (sKITL). To examine hematopoietic expansion following ex vivo coculture, we quantified phenotypic CD45.2 $2^{+}$ hematopoietic cells and CD 45.2 $2^{+}$lineage ${ }^{-} \mathrm{CIT}^{+} \mathrm{SCA} 1^{+}$HSPCs. Aged ECs promoted an increase in total CD $45.2^{+}$hematopoietic cell expansion in both young and aged HSPC cocultures when compared with their young EC counterparts (Figure 3A). Within this cell population, aged ECs promoted a significant increase in phenotypic aged HSPC expansion, while young HSPCs displayed no observable differences when compared with young EC feeders (Figure 3B and Supplemental Figure 1, A and B; supplemental material available online with this article; https://doi.org/10.1172/ jci.insight.93940DS1). To examine the long-term repopulating capacity of young and aged HSPCs cultured on young and aged ECs following 14 days of ex vivo expansion, total cocultured cells were collected and CD $45.2^{+}$hematopoietic cells were purified from EC feeders and competitively transplanted at a 1:1 ratio with young (3 months old) CD 45.1 ${ }^{+}$WBM cells into lethally irradiated young (3 months old) CD45.1 recipient mice. Mice were assessed for long-term multilineage engraftment 4 months after transplantation. To control for young and aged hematopoietic phenotypes, pre-expansion WBM input was competitively transplanted at a 1:1 ratio and analyzed at 4 months to confirm hematopoietic engraftment deficiencies and myeloid-biased output associated with aging (Figure 3, C-F). Young HSPCs cocultured on aged ECs showed a significant decrease in engraftment when compared with young HSPCs cocultured on young ECs (Figure 3C and Supplemental Figure 1C). Moreover, we observed that aged HSPCs cocultured on aged ECs were unable to competitively engraft in lethally irradiated animals, while aged HSPCs cocultured on 
A
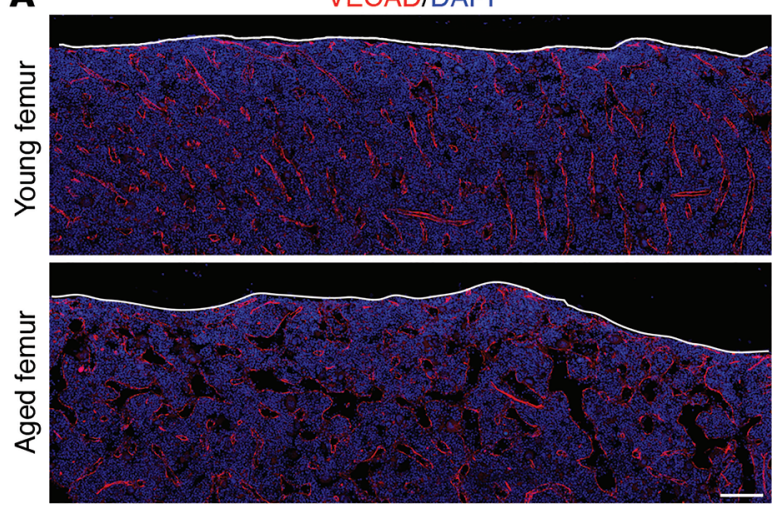

C

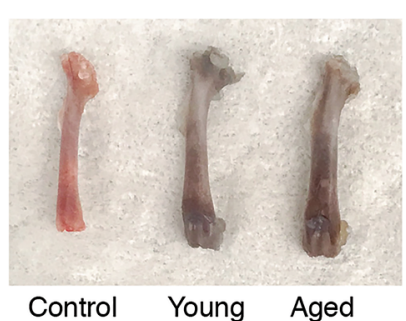

D

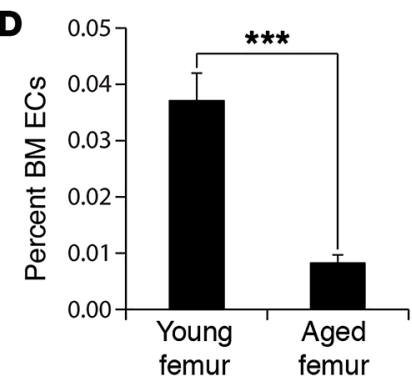

B
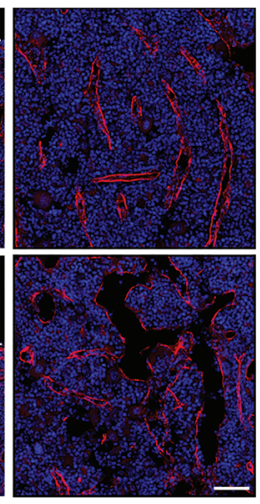

E

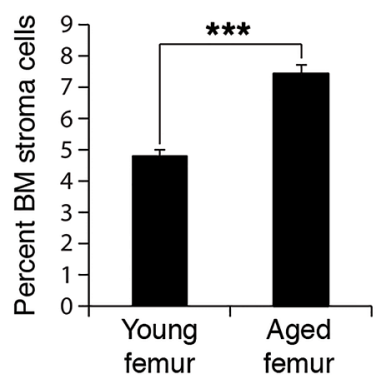

$\mathbf{F}$

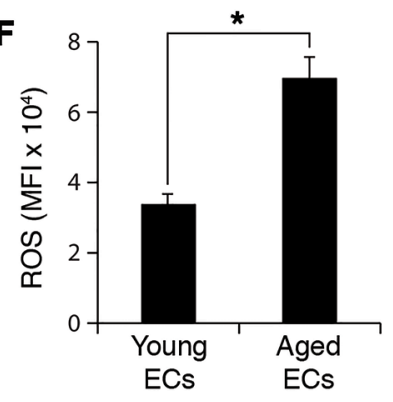

H

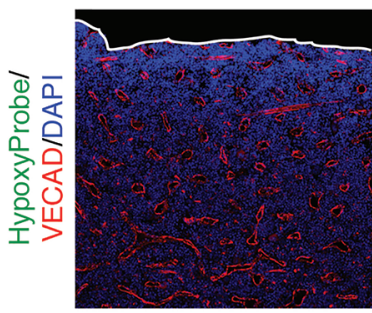

Young femur

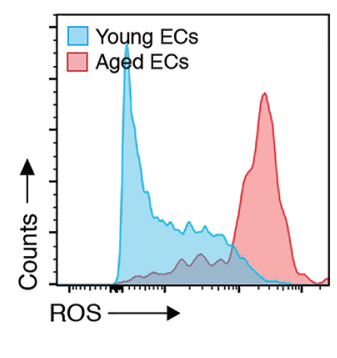

G

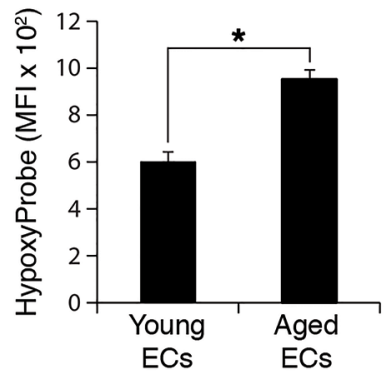

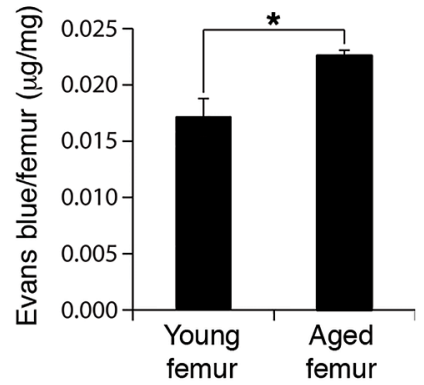

femur femur

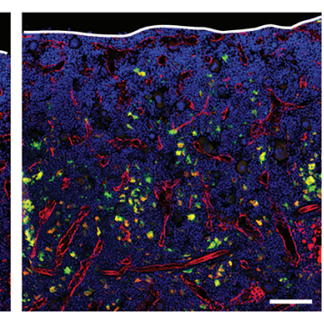

Aged femur

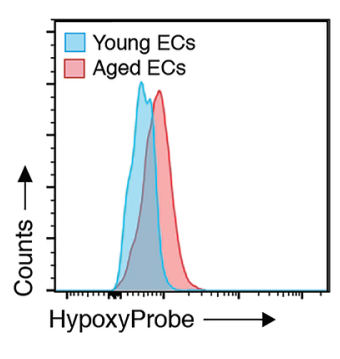

Figure 1. Aged BM vasculature displays functional alterations in vivo. (A) Representative longitudinal and inset images of femurs intravitally labeled with a vascular-specific VECAD antibody (red), showing morphological alterations in aged vasculature (white line demarcates cortical bone). Scale bars: $100 \mu \mathrm{m}$ (longitudinal images) and $50 \mu \mathrm{m}$ (insets). (B and C) Analysis of BM vascular leakiness in young and aged femurs. (B) Quantification of Evans blue dye extravasation ( $n=5$ mice/ cohort). (C) Representative femurs injected with Evans blue dye. Noninjected controls were used to determine baselines ( $n=5$ mice/cohort). (D and E) Frequency of recoverable (D) VECAD+CD31+CD45-TER119-BM ECs and (E) VECAD-CD31-CD45-TER119- stroma in young and aged femurs ( $n=5$ mice/cohort). (F) Quantification of mean fluorescence intensity (MFI) and representative histogram of ROS in VECAD+CD31+CD45-TER119- ECs from young and aged femurs showing an increase in ROS in aged ECs ( $n=3$ mice/cohort). (C) MFI quantification and representative histogram of pimonidazole adducts as detected by an anti-pimonidazole antibody (HypoxyProbe) in VECAD+CD31+CD45-TER119- ECs from young and aged femurs, demonstrating an increased hypoxia state in aged ECs ( $n=3$ mice/cohort). (H) Representative immunofluorescence images of HypoxyProbe-stained young and aged femurs, showing local changes in hypoxia (white line demarcates cortical bone). Scale bar: $50 \mu \mathrm{m}$. Error bars represent the sample mean \pm SEM. ${ }^{*} P<0.05$ and ${ }^{* *} P<0.001$, by unpaired, 2 -tailed Student's $t$ test.

young ECs were partially rejuvenated and displayed robust hematopoietic engraftment (Figure 3C and Supplemental Figure 1C). Taken together, these data suggest that aged ECs are significantly impaired in their HSC-instructive function. Despite the observed increase in engraftment of aged HSPCs cocultured on young ECs,

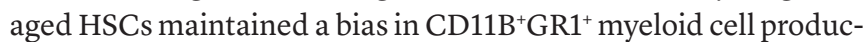
tion at the expense of $\mathrm{B}^{2} 2 \mathrm{O}^{+} \mathrm{CD} 19^{+} \mathrm{B}$ cells and $\mathrm{CD} 8^{+} / \mathrm{CD} 4^{+} \mathrm{T}$ cells when compared with young HSPC-young EC cocultured controls 
A
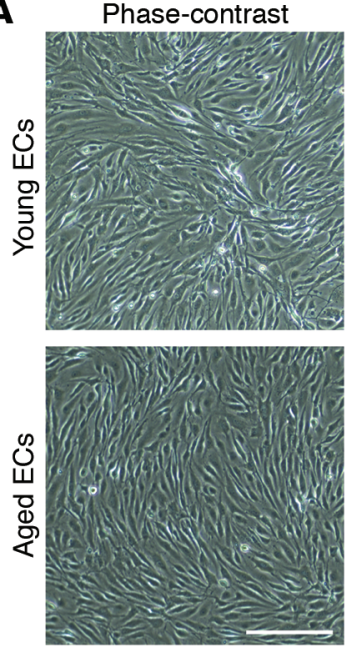

D

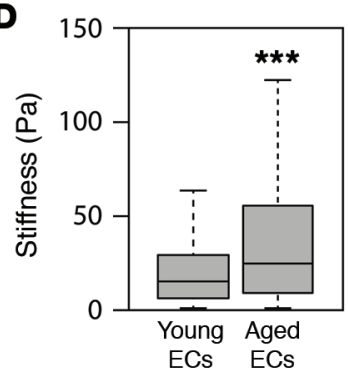

VECAD/DAP
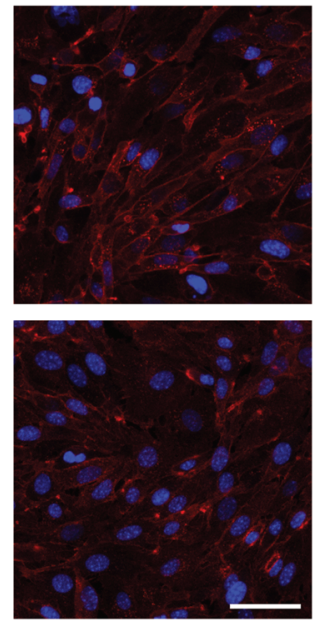

E

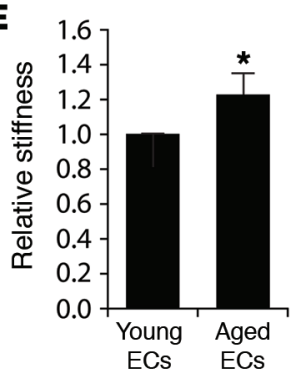

B
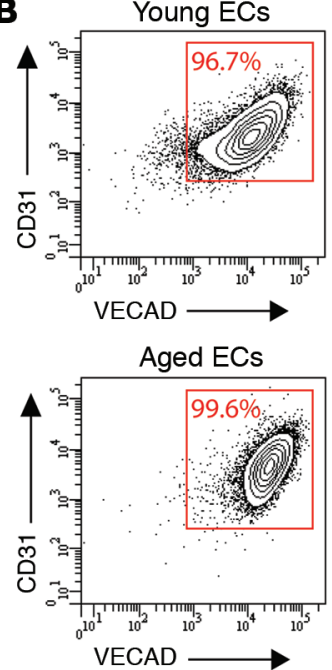

$\mathbf{F}$

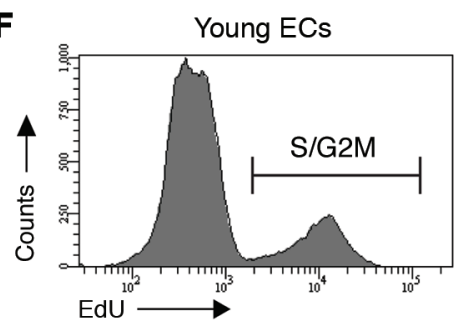

C Young ECs
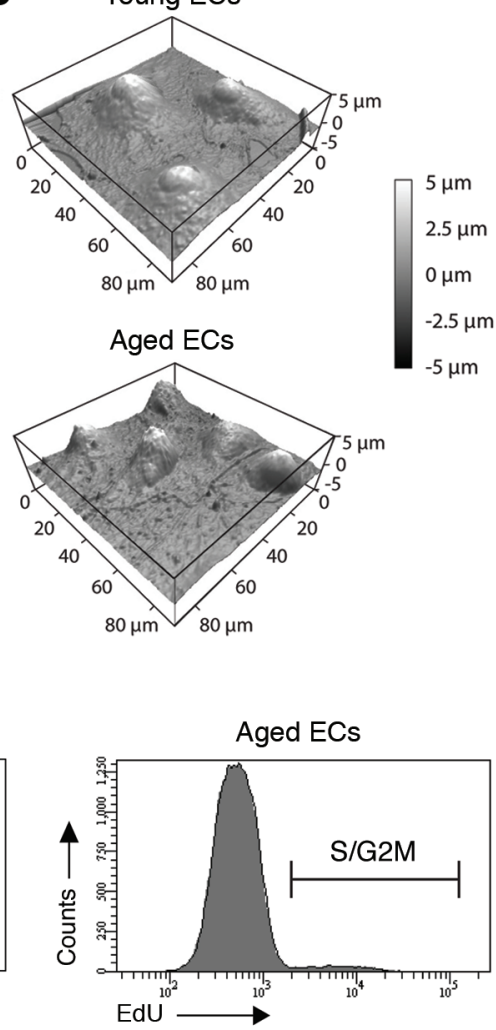

G

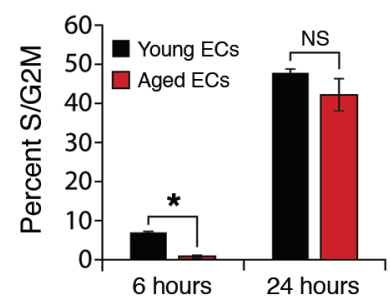

H

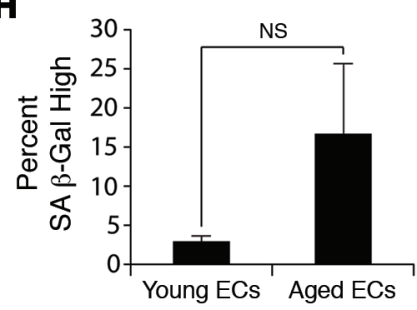

I
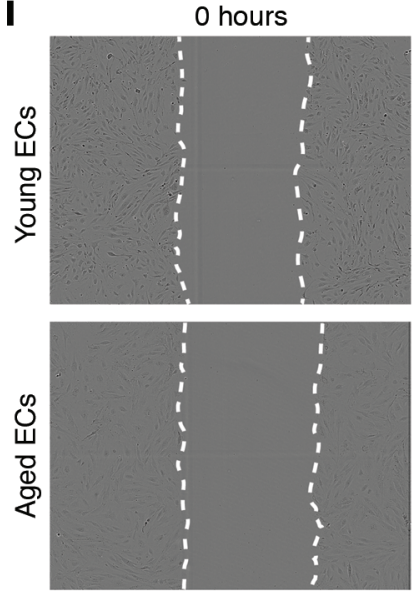

24 hours
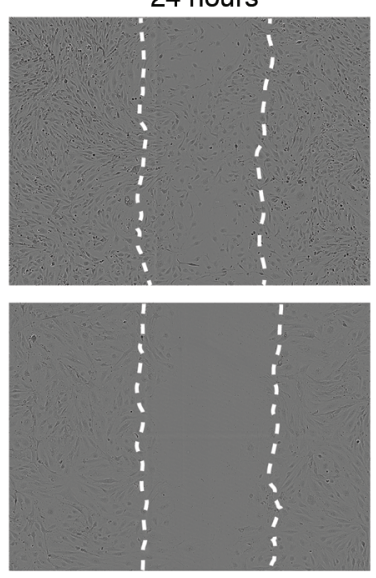

48 hours
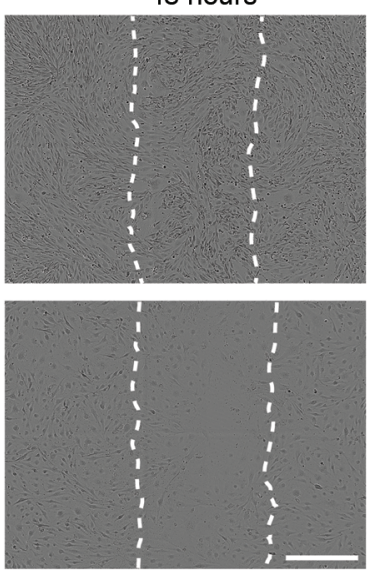

J

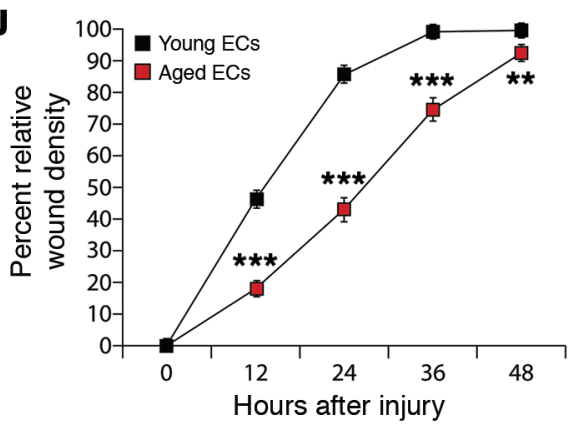

$\mathbf{K}$

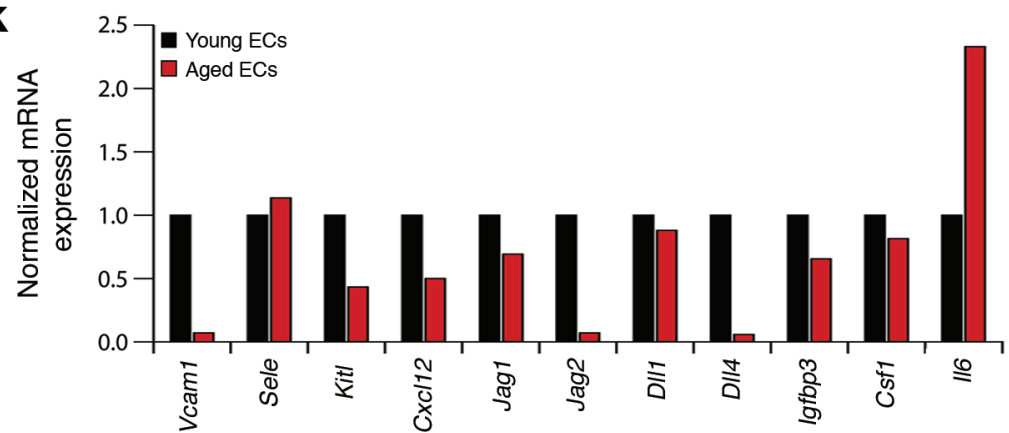


Figure 2. Characterization of cultured ECs from aged mice. (A) Representative phase-contrast and immunofluorescence images of cultured BMderived ECs from young and aged mice. Scale bars: $200 \mu \mathrm{m}$ (phase-contrast) and $50 \mu \mathrm{m}$ (immunofluorescence). (B) Representative flow plots of cultured ECs stained for VECAD+CD31+ demonstrating highly purified EC populations. (C-E) AFM analysis of elasticity in cultured young and aged ECs showing an increase in aged EC stiffness. (C) Representative reconstructed images of EC monolayers. (D) Box plots of the median stiffness in cultured young and aged ECs ( $n=3$ biological replicates). (E) Normalized relative EC stiffness ( $n=3$ biological replicates). ( $\mathbf{F}$ and $\mathbf{G}$ ) Proliferation status of cultured young and aged ECs. (F) Representative histograms of Edu incorporation following cell-cycle synchronization. (G) Quantification of Edu incorporation demonstrating an early inhibition of cell-cycle entry into the $S$ phase in aged ECS that was resolved by 24 hours ( $n=3$ biological replicates). (H) Quantification of SA $\beta$-gal activity in young and aged ECs ( $n=3$ biological replicates). (I and J) In vitro scratch wound-healing assay showing a functional delay in cell migration in aged ECs. (I) Representative phase-contrast images (dashed lines demarcate the initial scratch wound). Scale bar: $400 \mu \mathrm{m}$. (J) Quantification of EC wound healing ( $n=3$ biological replicates). (K) Normalized gene expression in cultured young and aged ECs ( $n=3$ biological replicates). ${ }^{*} P<0.05,{ }^{* *} P<0.01$, and ${ }^{* * *} P<0.001$. Significance was determined using an unpaired, 2-tailed Student's $t$ test, with error bars representing the sample mean \pm SEM. A nonparametric, 1-sided Wilcoxon rank-sum test was used to compare median endothelial stiffness in $\mathbf{D}$. Data are presented as box plots, with whiskers representing an IQR of \pm 1 .5. Relative endothelial stiffness in $\mathbf{E}$ was normalized to young ECs and presented as a $95 \% \mathrm{CI}$.

(Figure 3, D-F, and Supplemental Figure 1, D-F). Conversely, we found that young HSPCs cocultured on aged ECs acquired a myeloid bias, with a decrease in $\mathrm{B}$ and $\mathrm{T}$ cell frequencies (Figure 3 , D-F, and Supplemental Figure 1, D-F). These data, along with the observed decline in engraftment of young HSPCs cocultured on aged ECs, suggest that aged vascular endothelium, independent of other stromal constituents, is sufficient to impart hematopoietic aging phenotypes on young HSCs. Moreover, these data also allow for the possibility that EC-specific intervention may provide a therapeutic opportunity to increase HSC function in the elderly population following hematopoietic injury.

Infusion of aged ECs promotes a myeloid bias in vivo. ECs generated from a number of hematopoietic and nonhematopoietic tissues have been used to demonstrate their therapeutic potential following irradiative hematopoietic insult $(37,44-46)$. We have previously shown that infusion of young niche-specific $\mathrm{BM}$-derived ECs into young recipients following radiation- and chemotherapy-mediated myeloablative injury promotes rapid and efficient regeneration of the hematopoietic system by reducing the duration of pancytopenias and safeguarding HSC function $(37,43)$. In ex vivo coculture experiments, aged ECs were unable to support young HSC-repopulating activity and imparted a myeloid bias at the expense of $\mathrm{B}$ and $\mathrm{T}$ cell output in mice with long-term engraftment (Figure 3, C-F). To further examine the aging-related impaired instructive function of aged ECs in an in vivo context, we infused $5 \times 10^{5}$ aged ECs into young C57BL/6 mice on 4 consecutive days following myelosuppressive total body irradiation (TBI) and assessed peripheral hematopoietic recovery weekly (Figure 4A). Young ECs were used as a positive control. It is important to note that in these experiments (Figure 4), EC infusions were done in the absence of exogenous HSCT and addressed only endogenous hematopoietic recovery in young mice following myelosuppressive injury. We observed that the infusion of young ECs into young mice following myelosuppressive insult promoted rapid recovery of the hematopoietic system (WBC, RBC, and platelet counts), while the infusion of aged ECs was unable to support efficient hematopoietic recovery in young recipients (Figure $4 \mathrm{~B}$ ), suggesting that aged ECs have an impaired hematopoietic-instructive function in vivo. After allowing 28 days for recovery, we next examined the HSC-protective potential of EC infusions in these mice. WBM isolated from EC-infused young cohorts (CD45.2 $2^{+}$donor) was transplanted with a competitive dose of freshly isolated young CD45.1 ${ }^{+} \mathrm{WBM}$ at a 2:1 ratio into lethally irradiated young CD45.1 recipients (Figure 4A). We also performed competitive transplantation of WBM (CD45.2 $\left.{ }^{+}\right)$ isolated from steady-state young and aged mice (nonirradiated and without EC infusion) to serve as baseline controls for comparison of aging-induced hematopoietic phenotypes (Figure 4D and Supplemental Figure 2). We analyzed long-term, multilineage engraftment 4 months after transplantation. In agreement with ex vivo HSPC expansions (Figure 3C), we found that infusion of aged ECs was unable to support endogenous HSC activity in young mice (Figure 4E). Interestingly, infusion of aged ECs

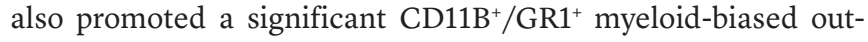
put at the expense of $\mathrm{B}^{2} 2 \mathrm{O}^{+} \mathrm{B}$ cell and $\mathrm{CD}^{+} \mathrm{T}$ cell engraftment when compared with young EC-infused animals (Figure 4E). Collectively, these data demonstrate that infusion of aged ECs into young recipients following myelosuppressive injury impairs endogenous HSC activity and imparts a myeloid bias, further supporting the idea that an aged vascular niche can impart aged hematopoietic phenotypes on a young hematopoietic system.

Young ECs enhance endogenous HSC activity in aged mice. Given that the elderly population responds poorly to the intensive myelosuppressive therapy that is often required to treat hematopoietic malignancies (47), we set out to test whether infusion of young ECs into aged recipients could provide therapeutic benefit by diminishing the duration of cytopenias following myelosuppressive irradiation. As observed in the young cohorts, aged mice receiving infusions of young ECs following myelosuppressive TBI had increased recovery of $\mathrm{WBM}, \mathrm{RBC}$, and platelet cell counts in the peripheral blood (PB), while infusions of aged ECs failed to promote improvement (Figure 4C). We next examined the HSC-rejuvenating potential of infusion of young ECs into aged mice following TBI-mediated myelosuppression. As previously described, WBM isolated from EC-infused aged cohorts (CD $45.2^{+}$donor) was transplanted 28 days after irradiation with a competitive dose of young CD45.1 $1^{+}$ WBM at a 2:1 ratio into lethally irradiated young CD45.1 recipients (Figure 4A). We analyzed the long-term, multilineage engraftment 4 months after transplantation. As previously observed in aged HSPC-young EC cocultures (Figure 3C), aged mice that received young ECs also demonstrated a marked improvement in overall hematopoietic engraftment (Figure 4F). Moreover, while infusions of young ECs could not resolve the myeloid-biased output of aged HSCs, young ECs were able to significantly increase the output of the $\mathrm{B}$ and $\mathrm{T}$ cell lineages in recipients (Figure $4 \mathrm{~F}$ ). Taken together, these data suggest that not only can young ECs be used to rejuvenate the functional output of endogenous aged hematopoietic cells in vivo, but they could also potentially be used to enhance exogenous hematopoietic engraftment following myeloablative preconditioning and $\mathrm{BM}$ transplantation. 
A

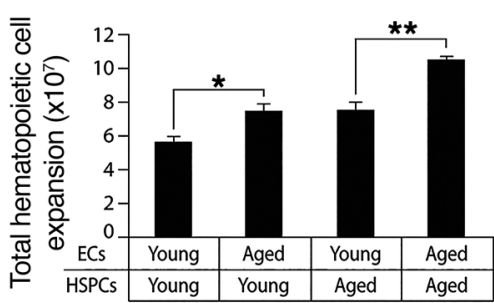

B

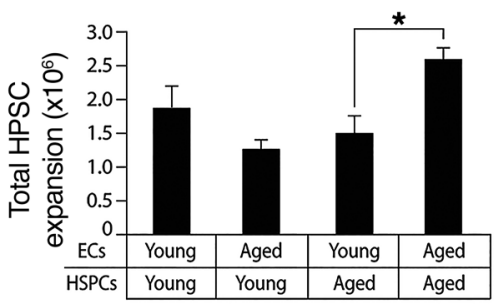

14-day ex vivo expansion (serum-free + 50 ng/ml sKITL)

C

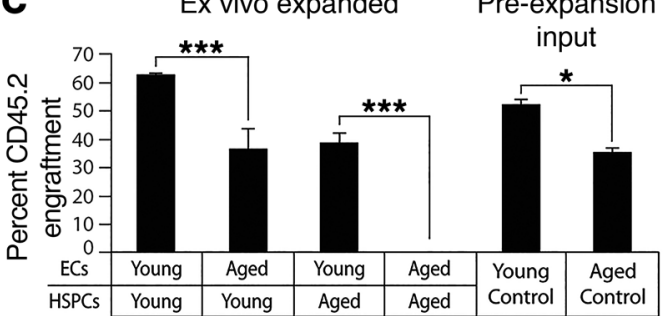

1:1 competitive transplantation (4 mo after Tpx)
D

Ex vivo expanded Pre-expansion input

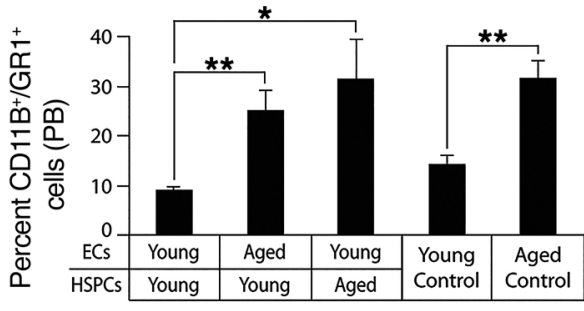

E

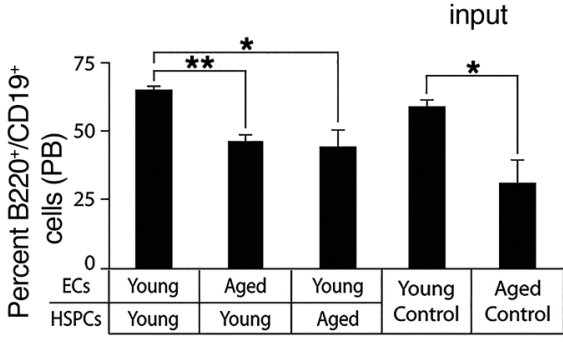

$\boldsymbol{F}$

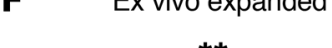

Pre-expansion

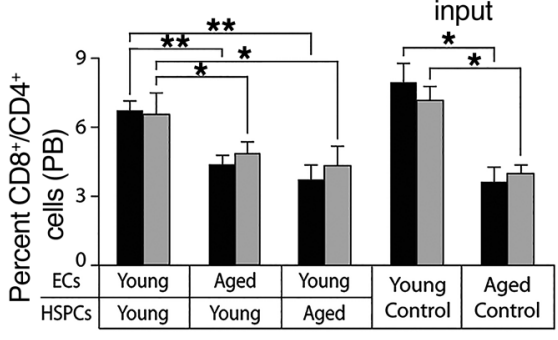

Lineage composition (4 mo after Tpx)

Figure 3. Aged ECs are sufficient to induce aged hematopoietic phenotypes. (A and B) Quantification of hematopoietic expansion by flow cytometry. (A) Total phenotypic CD45 hematopoietic cells and (B) CD45+lineage- ${ }^{-}$KIT $+5 C A 1^{+}$HSPCs ( $n=3$ independent cocultures). (C) Quantification of CD45.2 ${ }^{+}$ donor chimerism in PB 4 months after transplantation (Tpx), as measured by flow cytometry ( $n=5$ mice/cohort). Results show the ability of young ECs to restore hematopoietic engraftment of HSPCs following coculture, while aged ECs impaired young hematopoietic engraftment relative to that seen in age-matched coculture controls. Unmanipulated pre-expansion WBM cells from young or aged mice were competitively transplanted into lethally irradiated recipients to confirm age-dependent hematopoietic reconstitution phenotypes ( $n=5$ mice/cohort). (D-F) Quantification of donor-derived lineage ${ }^{+}$ hematopoietic repopulation 4 months after transplantation. Frequencies of (D) CD11B+CR1+ myeloid cells, (E) B220 ${ }^{+}$CD19+ B cells, and (F) CD8 $8^{+}($black)/ $\mathrm{CD}^{+}$(gray) T cell populations in PB were determined by flow cytometry. Young HSPCs cocultured with aged ECs acquired myeloid-biased engraftment at the expense of lymphopoiesis, while young ECs were unable to reverse the myeloid bias in aged HSPC expansions. Error bars represent the sample mean \pm SEM. ${ }^{*} P<0.05,{ }^{* *} P<0.01$, and ${ }^{* *} P<0.001$, by unpaired, 2 -tailed Student's $t$ test for like groupings.

Coinfusion of young ECs enhances HSCT and promotes overall survival. Allogeneic transplantation of adult BM can provide a cure for patients with life-threatening hematological disorders. However, there is a large group of patients who do not have access to HLA-matched BM. Strategies to expand the number of patients who can use a single BM harvest by minimizing the number of BM cells necessary for rapid hematopoietic recovery and long-term, multilineage engraftment would be useful. Additionally, there are hematopoietic disorders, such as sickle cell disease, that could benefit from autologous transplantation of the patient's own gene-corrected $\mathrm{BM}$. However, many cases result in BM cell numbers that are suboptimal for achieving robust engraftment. To this end, we set out to determine whether a single-dose coinfusion of young ECs in the context of a limiting HSCT cell dose could enhance hematopoietic engraftment and promote survival. To this end, we transplanted $10^{5}$ young or aged WBM cells into young recipients, with or without a single coinfusion of $5 \times 10^{5}$ young ECs, following a lethal dose of TBI (9.50 Gy). Of note, transplantation of $2 \times$ $10^{5}$ young WBM cells alone into recipient mice results in complete radioprotection following lethal irradiation, while a $10^{5} \mathrm{WBM}$ cell dose is suboptimal. We used steady-state (nonirradiated and without WBM or EC infusion), young ECs alone, or PBS infused cohorts to establish baselines. Survival curves revealed that a single dose of $5 \times 10^{5}$ young ECs alone was unable to radioprotect mice following myeloablative injury (Figure $5 \mathrm{~A}$ ). Infusion of $10^{5} \mathrm{WBM}$ cells from either young or aged donors resulted in $70 \%$ and $50 \%$ survival rates, respectively, for recipient mice (Figure 5, A and B). However, mice that were coinfused with $10^{5}$ young WBM cells and $5 \times 10^{5}$ young ECs had a $100 \%$ survival rate (Figure $5 \mathrm{~A}$ ). Moreover, animals coinfused with $10^{5}$ aged WBM cells and $5 \times 10^{5}$ young ECs achieved $90 \%$ survival rates (Figure $5 \mathrm{~B}$ ). These data demonstrate that coinfusion of young ECs with limited numbers of WBM from both young and aged donors results in increased survival following myeloablation, with all mice achieving long-term, multilineage engraftment (Supplemental Figure 3, A and B). Furthermore, coinfusion of young ECs and young WBM into lethally irradiated mice also improved short-term and long-term hematopoietic recovery when compared with the young WBM-alone cohort (Figure 5C), while young ECs coinfused with aged WBM resulted in a minimal increase in recovery of RBC and platelets as compared with infusion of aged WBM alone (Figure 5D). We next examined whether young ECs conferred a radioprotective effect on coinfused HSPC activity by assessing splenic CFU (CFU-S) (48) and found that the coinfusion of young ECs with either young or aged WBM resulted in an increase in the number of hematopoietic colonies that formed in the spleen following transplantation (Figure 5, E and F, and Supplemental Figure 4A). This observation supports our previous data (Figure 4, E and F) suggesting that infusions of young ECs following myelosuppressive TBI protects endogenous HSC activity. Therefore, we examined the possibility that coinfusion of 
A

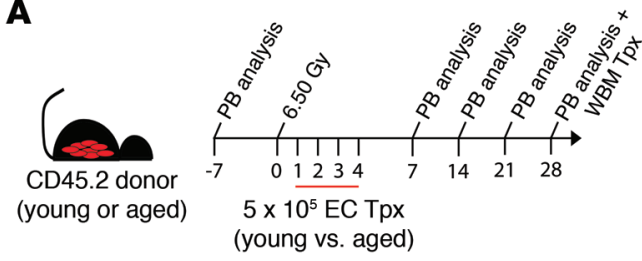
(young vs. aged)

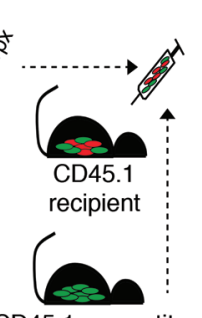

CD45.1 competitor
Transplantation strategy

$\left(2 \times 10^{6}\right.$ CD45.2 donor WBM $^{+}$

$+10^{6}$ CD45. 1 competitor WBM)

†Young or aged steady-state WBM donor

tPost-irradiation young WBM donor

(with young or aged EC Tpx)

${ }^{\dagger}$ Post-irradiation aged WBM donor (with young or aged EC Tpx)
B

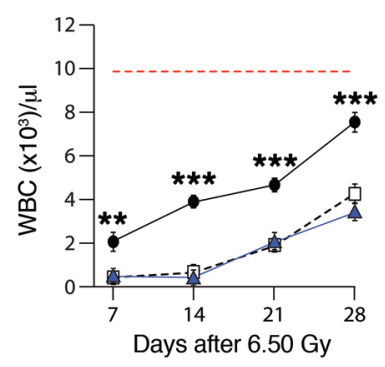

\section{Aged mice}

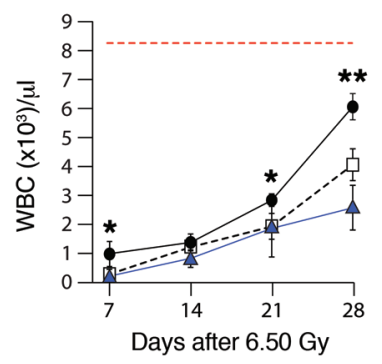

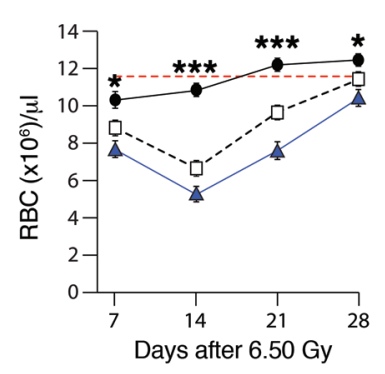

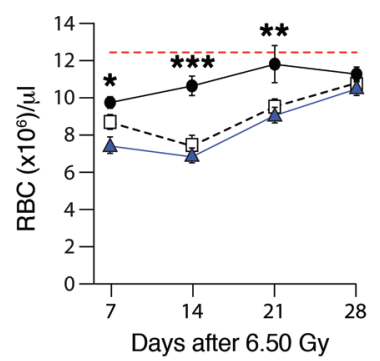

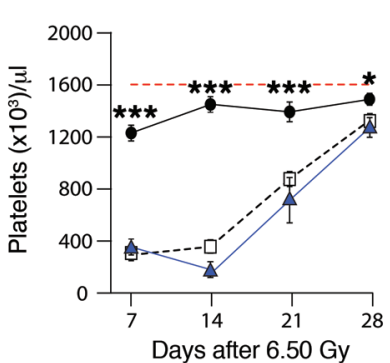

- - Steady state

$\square$ PBS control

- Young ECs

$\triangle$ Aged ECs
D Steady-state WBM donor
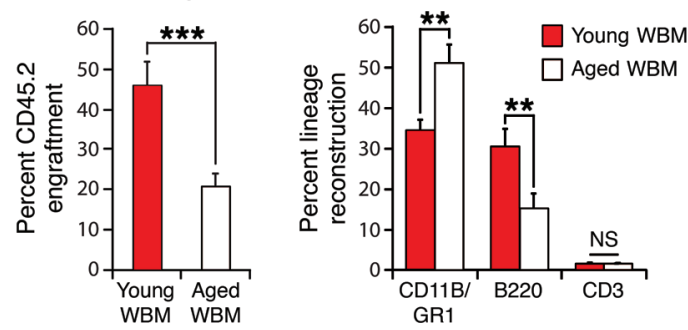

F Post-irradiation aged WBM donor
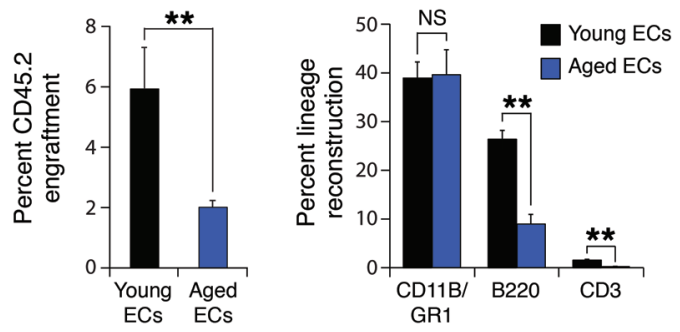

Figure 4. Infusion of young endothelium promotes hematopoietic recovery in aged recipients following myelosuppressive injury. (A) Schematic of the EC infusion strategy. (B and C) Time course of PB recovery of (B) young and (C) aged mice following irradiation (6.50 Gy) and infusion of either young ECs, aged ECs, or PBS vehicle control ( $n=5$ mice/cohort). The results demonstrated the myeloprotective effect of young EC transplantation following hematopoietic insult in both young and aged recipients, while the result with aged EC transplantation was indistinguishable from that observed in the PBS vehicle-infused controls. (D-F) Quantification of CD45.2+ donor chimerism and multilineage engraftment in PB 4 months after donor WBM transplantation as measured by flow cytometry ( $n=5$ mice/cohort). (D) Unmanipulated steady-state young and aged WBM was competitively transplanted to confirm reduced CD45.2 $2^{+}$hematopoietic engraftment and phenotypic CD11B ${ }^{+} / \mathrm{CR} 1^{+}$myeloid bias in the aged WBM transplantation cohort ( $n=5$ mice/cohort). (E) Young and (F) aged donors infused with young ECs demonstrated an increase in hematopoietic engraftment, while supporting an increase in $\mathrm{B22O}^{+}$and $\mathrm{CD}^{+}$lymphoid reconstitution. Error bars represent the sample mean \pm SEM. ${ }^{*} P<0.05,{ }^{* *} P<0.01$, and ${ }^{* * *} P<0.001$, by unpaired, 2 -tailed, Student's $t$ test for comparisons at individual time points. Steady-state and PBS controls were included as recovery reference points and were not included in the statistical analysis. 
A Young WBM Tpx

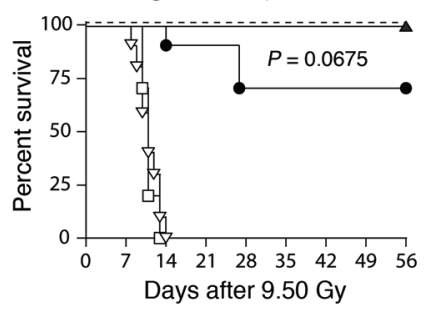

\section{Young WBM Tpx}

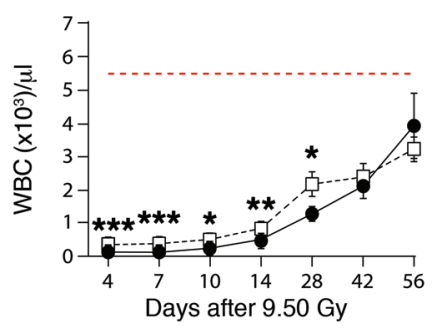

D Aged WBM Tpx
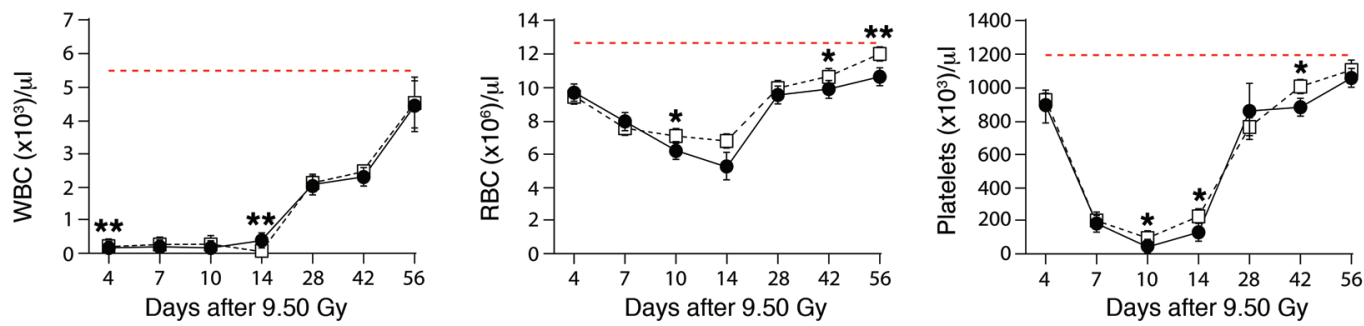

-- Steady state

- Aged WBM

$\square$ Aged WBM + young ECs
B Aged WBM Tpx
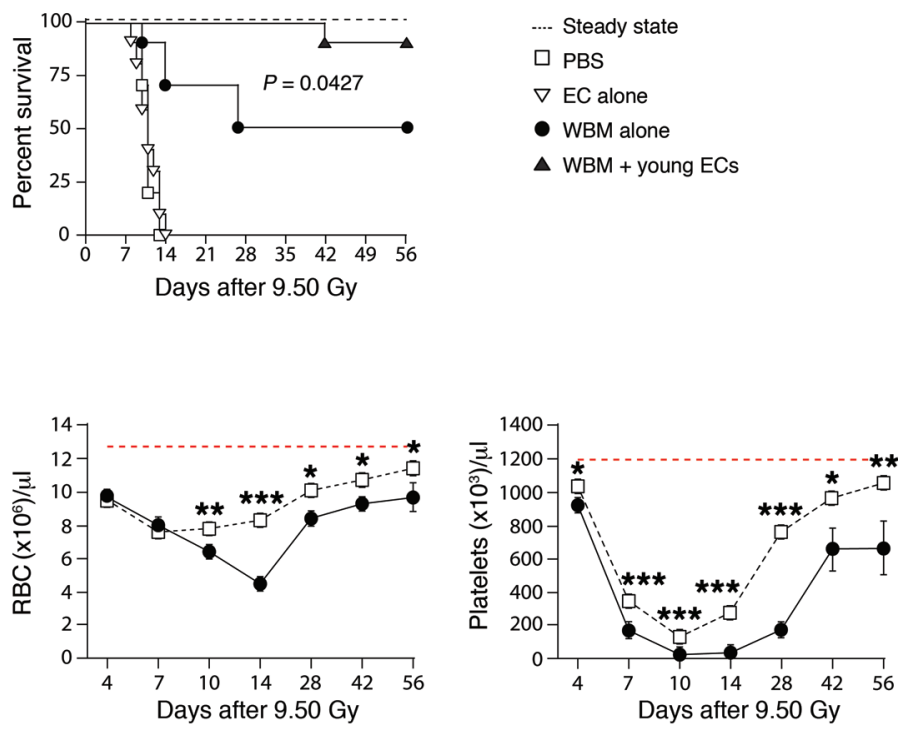

-- Steady state

- Young WBM

$\square$ Young WBM + young ECs
E

E
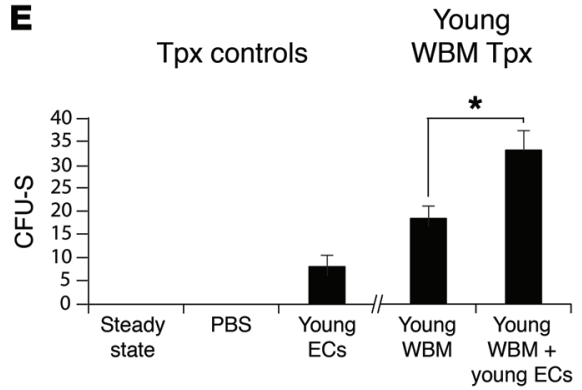

Figure 5. Coinfusion of young endothelium enhances BM transplantation. (A and B) Survival curves for mice transplanted with $10^{5}$ WBM cells from either (A) young or (B) aged animals, showing an increase in overall survival in WBM cohorts coinfused with ECs ( $n=10$ mice/cohort). Note: Data in $\mathbf{A}$ and $\mathbf{B}$ share the same steady-state, PBS, and EC-alone controls. The survival curve significance between the WBM and WBM-plus-EC cohorts was calculated using a log-rank test. (C and D) Time course of hematopoietic recovery in the PB of recipient mice coinfused with $10^{5}$ young or aged WBM cells, with or without young ECs. EC-coinfused animals had a significant increase in hematopoietic recovery ( $n=10$ mice/cohort). (E and F) Quantification of CFU-S in mice transplanted with $10^{5}$ WBM cells from $(\mathbf{E})$ young or $(\mathbf{F})$ aged donors, with or without young ECs, demonstrating an increase in hematopoietic progenitor activity in cohorts coinfused with ECs. CFU-S numbers were scored 8 days after irradiation ( $n \geq 5$ mice/cohort). (G and $\mathbf{H}$ ) Log-fraction plot of limiting dilution analysis showing the frequency of long-term multilineage repopulation of WBM from (G) young or $(\mathbf{H})$ aged mice transplanted into lethally irradiated recipients and coinfused or not with young ECs ( $n=10$ mice/cohort). Dashed lines indicate $95 \% \mathrm{Cls}$. Stem cell frequency and significance were determined using ELDA. Error bars represent the sample mean \pm SEM. ${ }^{*} P<0.05$, ${ }^{* *} P<0.01$, and ${ }^{* * *} P<0.001$, by unpaired, 2-tailed Student's $t$ test. Steady-state, $\mathrm{PBS}$, and $\mathrm{EC}$-alone controls were reference points and were not included in the statistical analysis.

young ECs with WBM could increase HSC-repopulating activity. We performed limiting-dilution analysis (LDA) by transplanting 2 $\times 10^{5}, 10^{5}$, and $5 \times 10^{4} \mathrm{WBM}$ cells isolated from steady-state young or aged mice, with or without a coinfusion of $5 \times 10^{5}$ young ECs, into lethally irradiated recipients. Survival was monitored for 6 months and used to quantify the nonresponding fraction. Young and aged WBM coinfused with young ECs showed a significant, approximately 2-fold (young WBM; $P=0.0225$ ) and approximately 3 -fold (aged WBM; $P=0.0035$ ) increase in hematopoietic repopulating activity when compared with WBM alone (Figure 5, $\mathrm{G}$ and $\mathrm{H}$, and Supplemental Figure 4, B and C). The increase in hematopoietic reconstitution was independent of homing defects 
A

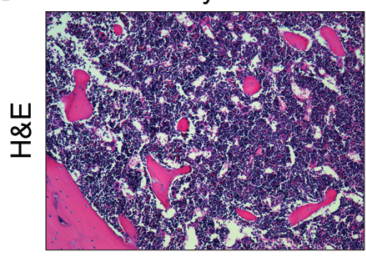

B

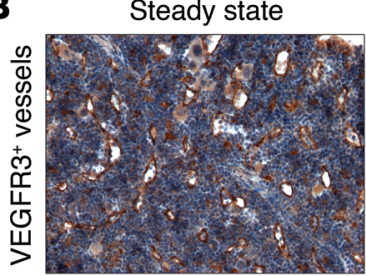

Young WBM

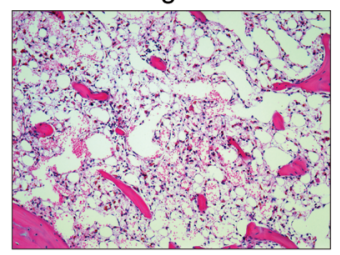

Day 7 after 9.50 Gy
Young WBM + ECs

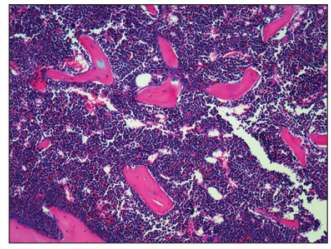

Young WBM + ECs

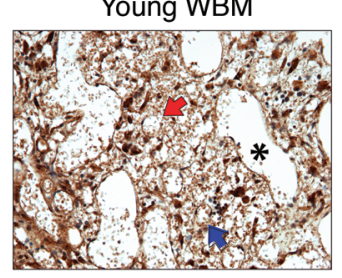

Day 7 after 9.50 Gy
Aged WBM

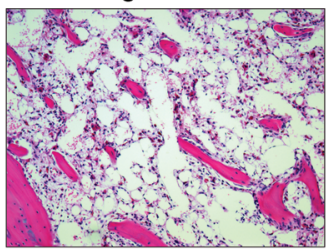

Day 7 after 9.50 Gy

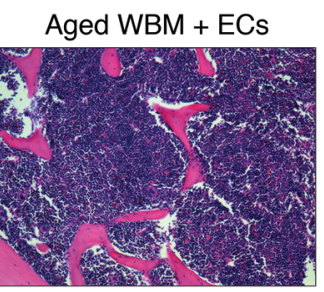

Aged WBM

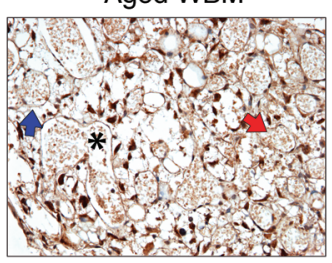

Day 7 after 9.50 Gy
C

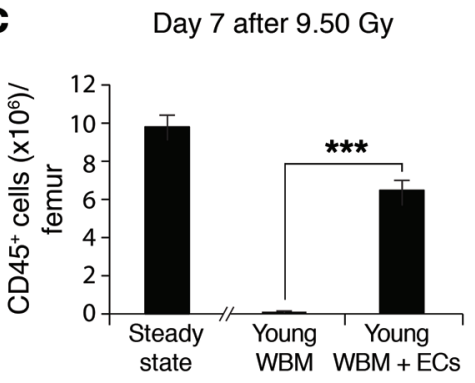

D

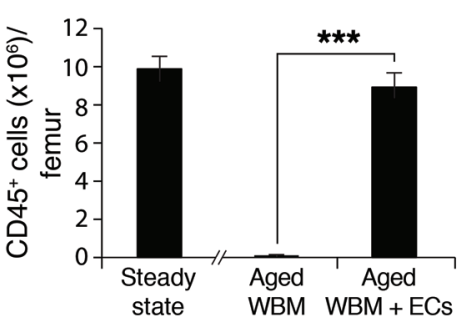

E Day 7 after 9.50 Gy

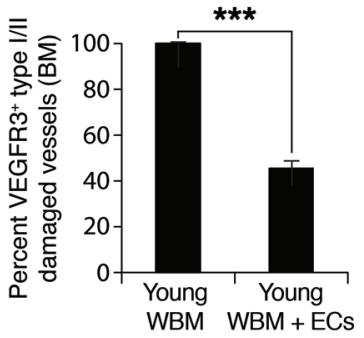

Aged WBM + ECs

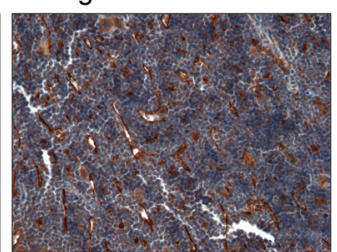

F Day 7 after 9.50 Gy

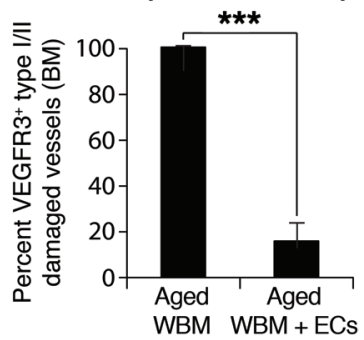

Figure 6. Young EC coinfusion radioprotects the BM microenvironment. Lethally irradiated ( $9.50 \mathrm{Cy}$ ) mice were coinfused with either $10^{5}$ young or aged WBM cells and $5 \times 10^{5}$ young BM ECs. (A) Representative H\&E-stained longitudinal femur sections from coinfused mice 7 days after irradiation ( $n=10$ mice/ cohort). Original magnification, $\times 100$. (B) Representative images of damaged VEGFR3 ${ }^{+}$femoral vessels, including type I hemorrhagic (asterisk), type I discontinuous (red arrow), and type II regressed (blue arrow), 7 days after irradiation, demonstrating radioprotection of the vascular niche ( $n=10$ mice/ cohort). Sections were counterstained with hematoxylin. Original magnification, $\times 200$. (C and D) Quantification of total BM CD45 cells showing mitigation of panhematopoietic injury in cohorts coinfused with young or aged WBM and young ECs ( $n=10$ mice/cohort; data are related to $\mathbf{A})$. (E and $\mathbf{F})$ Quantification of type I/II damaged VECFR3 ${ }^{+}$sinusoidal vessels in cohorts coinfused with young or aged WBM and young ECs ( $n=10$ mice/cohort; data are related to B). Error bars represent the sample mean \pm SEM. ${ }^{* *} P<0.001$, by unpaired, 2 -tailed Student's $t$ test. Steady-state controls were used as a reference point and were not included in the statistical analysis.

(Supplemental Figure 4, D and E). These data demonstrate that coinfusion of young ECs improves the overall survival of myeloablated recipients and enhances HSCT repopulating ability.

Coinfusion of young ECs radioprotects the BM sinusoidal vascular niche. Sinusoidal endothelium within the BM microenvironment undergoes significant damage in response to irradiation (49). Regeneration of BM sinusoidal ECs is also required for hematopoietic engraftment and regeneration following radiation-induced injury (50). The observed increase in HSC engraftment in coinfused cohorts suggests that infused ECs may protect the BM vascular microenvironment. Therefore, we examined BM recovery in EC-coinfused cohorts following myeloablative irradiation. Coinfusions of young ECs in young and aged WBM groups revealed a significant increase in BM cellularity and $\mathrm{CD} 45^{+}$hematopoietic cell frequency 7 days after irradiation (Figure 6, A, C, and D). Using a VEGFR3 antibody to distinguish BM sinusoidal endothelium, we next assessed the extent of vascular damage in coinfused mice. Young ECs mitigated BM sinusoidal endothelial damage, displaying a significant decrease in type I and type II endothelial damage (Figure 6, B, E, and F). Radioprotection of BM cellularity extended to early (day 4) and later (day 14) time points (Figure 7, A and B). Moreover, WBM-transplanted mice coinfused with young ECs had radioprotection of BM myeloid cells (Figure 7, C-F). Interestingly, a single dose of coinfused young ECs extended their regenerative effects to other radiosensitive tissues, including the spleen and intestine (Supplemental Figure 5), further expanding the therapeutic potential of infusions of young ECs to mitigate damage caused by radiation-mediated injury.

\section{Discussion}

Understanding the aging-dependent cellular changes to the BM microenvironment that impair HSC function and hematopoietic output will be critical in developing new and adjunctive therapies to treat age-related hematological disorders. Here, we have shown that physiological aging of ECs is sufficient to promote functional aging of young HSCs in ex vivo coculture experiments (Figure 3, $\mathrm{C}-\mathrm{F})$. We confirmed these ex vivo results in an in vivo model system in which aged ECs infused into young mice following myelo- 
A

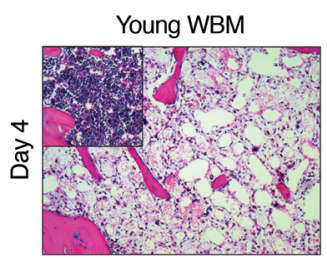

Young WBM + ECs

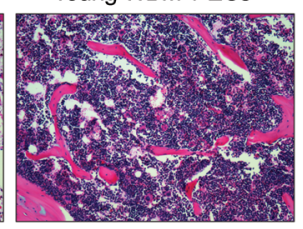

กั
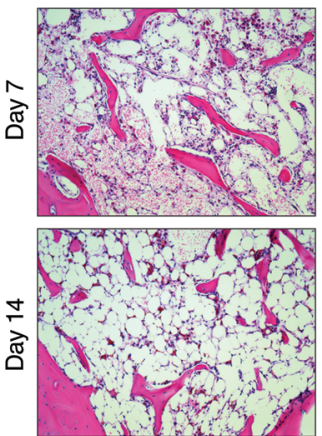

B
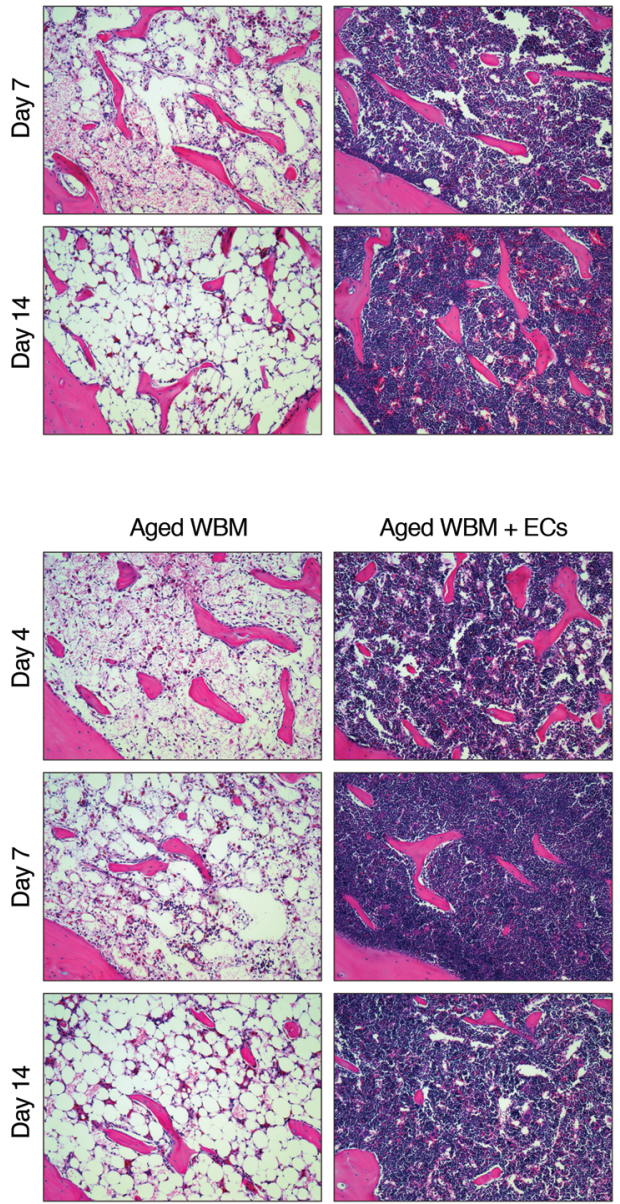

Aged WBM + ECs
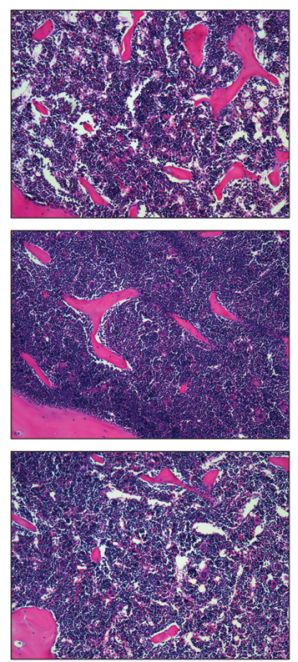

C

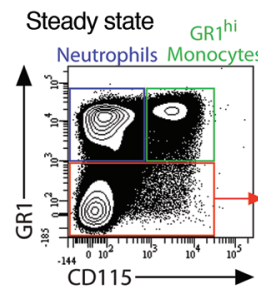

Young WBM

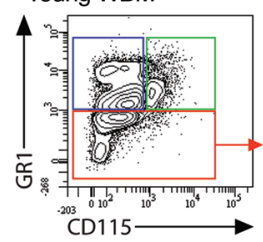

$$
\mathrm{CD} 115 \stackrel{10^{3}}{\longrightarrow}
$$

Young WBM + ECS
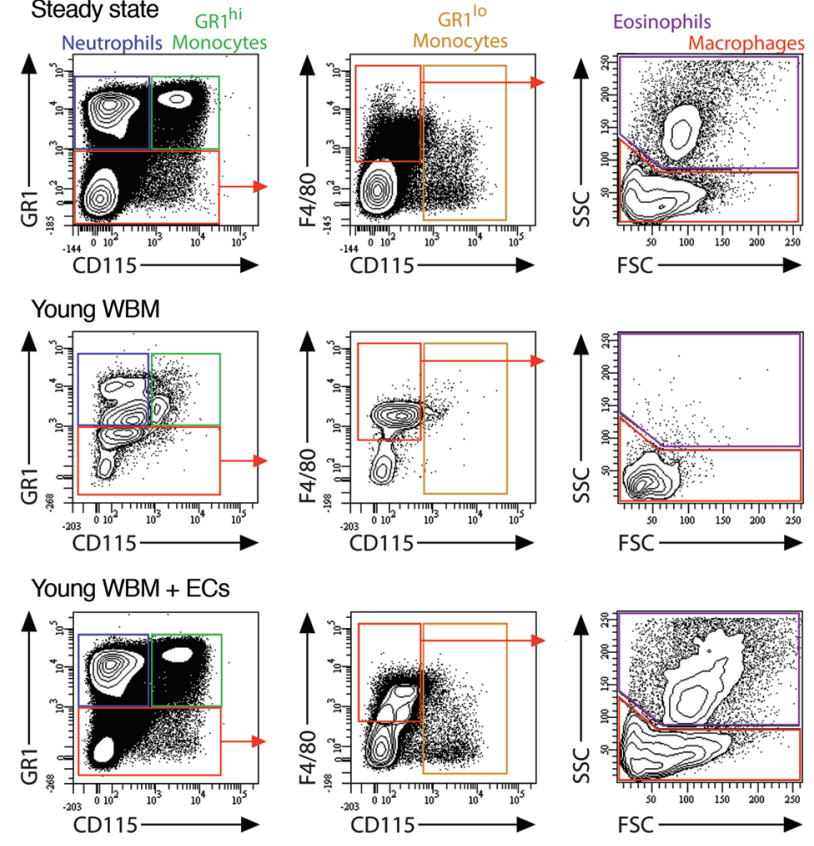

D
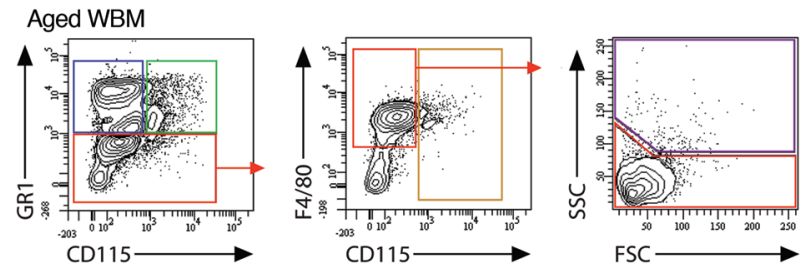

Aged WBM + ECs
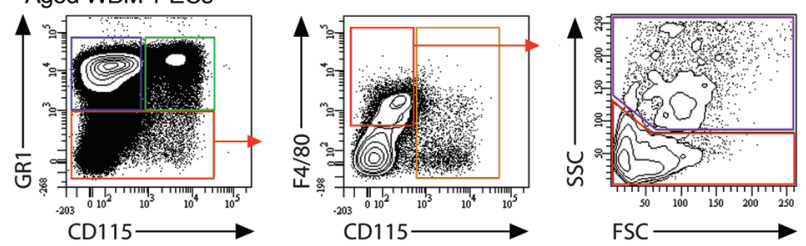
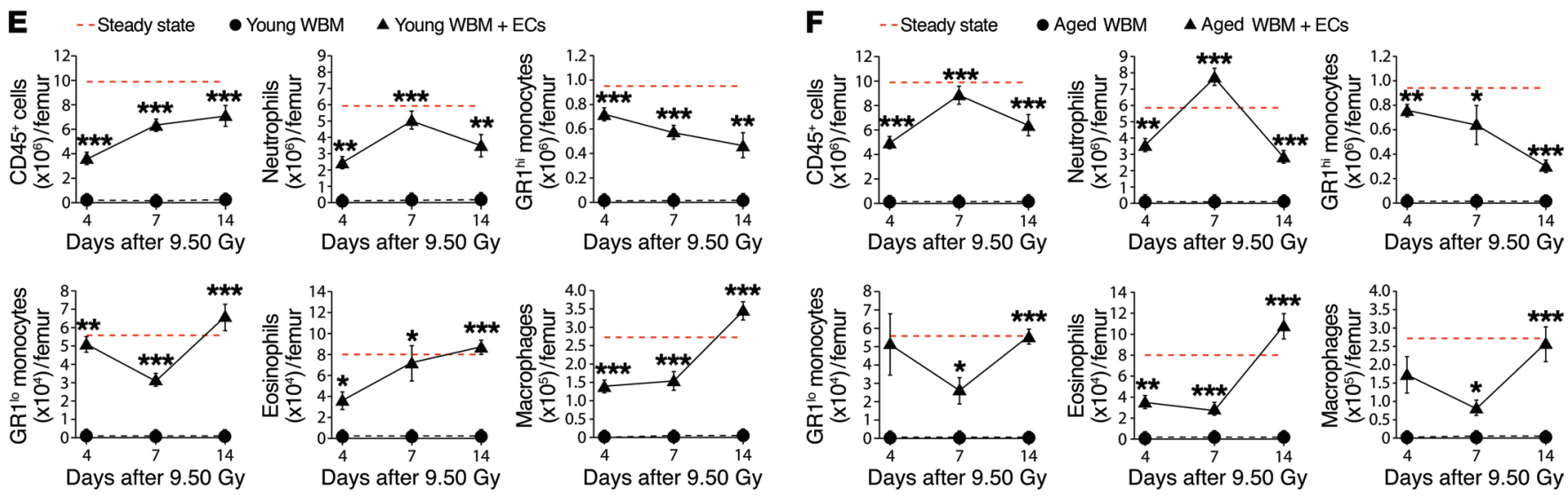

Figure 7. Young EC coinfusion mitigates myeloablative hematopoietic injury. (A and B) Representative H\&E-stained longitudinal femur time course after irradiation (9.50 Gy) for mice coinfused with (A) young and (B) aged WBM plus ECs ( $n=10$ mice/cohort). Original magnification, $\times 100$. Steady-state control H\&E-stained sections are shown in the inset. (C and $\mathbf{D})$ Representative contour plots of BM GM frequency in (C) young and (D) aged WBM-EC coinfusions after irradiation. (E and $\mathbf{F}$ ) Time course of BM GM counts in (E) young and (F) aged WBM-EC coinfusions 7 days after irradiation. ${ }^{*} P<0.05$, ${ }^{*} P<0.01$, and ${ }^{* * *} P<0.001$, by unpaired, 2-tailed Student's $t$ test, for comparisons of WBM with WBM-EC coinfusion cohorts at individual time points. Steady-state controls were used as a reference point and were not included in the statistical analysis. FSC, forward scatter; SSC, side scatter. 
suppressive irradiation imparted a myeloid bias at the expense of $\mathrm{B}$ and $\mathrm{T}$ cell output, while inhibiting HSC engraftment (Figure 4E). These observations were independent of aging-related changes to other microenvironmental cellular constituents, suggesting that aged endothelium is sufficient to drive aging-related hematopoietic defects. These results align with data demonstrating that an aged microenvironment can lead to aging-related hematopoietic outcomes (26), including reduced hematopoietic clonality (28) and myeloid skewing through the inflammatory cytokine Rantes (also known as CCL5) (51). While the decrease in expression of the verified pro-HSC factors Kitl, Cxcl12, and NOTCH ligands in aged ECs likely contributes to their impaired HSC-supportive function (Figure $2 \mathrm{~K}$ ), there are probably a number of additional pathways and factors that contribute to the observed EC-mediated aging phenotypes acquired in young HSCs. To our knowledge, this is the first evidence to demonstrate that aged endothelium can directly initiate age-related hematopoietic phenotypes, allowing for potential therapeutic intervention in aged vascular niches.

Our group and others have previously demonstrated the utility of ECs in enhancing hematopoietic recovery and safeguarding HSPCs following irradiation $(37,43-46,52)$. In these experiments, repeated i.v. EC doses or implanted donor vasculature provides a significant hematopoietic radioprotective effect in young mice. However, it is not clear whether this protective effect extends to an aged hematopoietic system $(11,15)$. Coculture of aged HSPCs on young ECs could partially rejuvenate HSC activity, but failed to reverse the aging-related intrinsic myeloid bias (Figure 3, C-F). While infusion of young ECs into aged mice following sublethal myelosuppressive irradiation was also able to significantly enhance peripheral hematopoietic recovery and endogenous aged HSC activity (Figure 4, C and F), it was unable to revert the observed myeloid bias. Infusions of young ECs were able to enhance $\mathrm{B}$ and $\mathrm{T}$ cell output in aged mice (Figure $4 \mathrm{~F}$ ), further demonstrating their therapeutic potential. Following a lethal dose of ionizing TBI, a single coinfusion of young ECs was able to significantly increase survival, hematopoietic progenitor activity, and HSC engraftment in mice transplanted with aged WBM (Figure 5, $\mathrm{B}, \mathrm{F}$, and $\mathrm{H})$. Hematopoietic engraftment and reconstitution are dependent on the successful regeneration of BM sinusoidal endothelium following irradiation (50). EC coinfusion significantly diminished VEGFR3 ${ }^{+}$sinusoidal EC damage by decreasing the prevalence of type I and type II regressed and hemorrhagic vessels within the BM (Figure 6, B, E, and F), preserved BM cellularity (Figure 6, A, C, and D, and Figure 7, A and B), and enhanced BM granulocyte-macrophage (GM) populations (Figure 7, E and F). These results demonstrate that young ECs can act as a supportive cellular therapy, protecting the aged BM microenvironment following radiation-mediated injury and promoting the rejuvenation of an aged hematopoietic system.

Altogether, our data demonstrate that a young BM vascular niche plays a critical role in supporting homeostatic hematopoiesis and HSC activity and that physiological aging disrupts endothelium-instructive function. This suggests that, by providing young endothelium via infusions, we can augment current therapies that rely on curative myeloablative strategies to enhance hematopoietic outcomes, including overall HSC engraftment efficiency, restoration of $\mathrm{B}$ and $\mathrm{T}$ cell frequencies, and overall survival. This study provides additional ex vivo and in vivo tools to dissect the molecular changes in aged ECs that play a role in modulating HSC activity during physiological aging and elucidate the mechanisms by which dysregulation of the homeostatic BM vascular microenvironment leads to many common age-related hematopoietic disorders.

\section{Methods}

Mice. Young and aged C57BL/6 (CD45.2) mice were purchased from the National Institute on Aging and from Taconic Biosciences. B6.SJL-Ptprc ${ }^{a} P e p c^{b}$ /BoyJ (CD45.1) mice were purchased from The Jackson Laboratory. All animals were maintained in specific pathogen-free housing. For hematopoietic recovery, reconstitution, and EC transplantation studies, mice were subjected to total body $\gamma$-irradiation $\left({ }^{137} \mathrm{Cs}\right) 24$ hours prior to transplantation. Transplant recipients were given PicoLab Mouse 20 antibiotic feed (0.025\% trimethoprim and $0.124 \%$ sulfamethoxazole; LabDiet) 24 hours prior to irradiation and subsequently maintained for 28 days.

Femur IHC. Young (3 months old) and aged (24 months old) mice were intravitally labeled for 10 minutes with $25 \mu \mathrm{g}$ Alexa Fluor 647conjugated VECAD antibody (BV13; BioLegend) via retro-orbital injection. Following labeling, mice were sacrificed and perfused via intracardiac injection of PBS (pH 7.2). Femurs were fixed overnight in $4 \%$ paraformaldehyde (PFA) in PBS (pH 7.2), decalcified in 10\% EDTA for 72 hours at room temperature, cryopreserved in 30\% sucrose for 48 hours at $4^{\circ} \mathrm{C}$, and embedded in 50\% OCT (Tissue-Tek) and 50\% sucrose. Longitudinal femur sections $(12-\mu \mathrm{m}$ thickness) were cut using a CM 3050 S Cryostat (Leica), counterstained with $1 \mu \mathrm{g} / \mathrm{ml}$ DAPI (BioLegend), and mounted using Prolong Gold Anti-fade Solution (Life Technologies, Thermo Fisher Scientific). Femur sections were imaged on an LSM 710 confocal microscope (Zeiss).

Flow cytometry and cell sorting. Before cell staining, $\mathrm{F}_{\mathrm{c}}$ receptors were blocked with a CD16/32 antibody (clone 93; BioLegend) in PBS (pH 7.2) containing 0.5\% BSA (fraction V) and 2 mM EDTA for 10 minutes at $4^{\circ} \mathrm{C}$. $\mathrm{F}$-blocked samples were stained in PBS (pH 7.2) containing 0.5\% BSA (fraction $\mathrm{V}$ ) and $2 \mathrm{mM}$ EDTA for 30 minutes at $4^{\circ} \mathrm{C}$ with fluorochrome-conjugated antibodies, according to the manufacturer's protocol. Stained cells were washed in PBS (pH 7.2) containing $0.5 \%$ BSA (fraction V) and $2 \mathrm{mM}$ EDTA and fixed in $1 \%$ PFA in PBS (pH 7.2) with 2 mM EDTA for flow analysis, or resuspended in PBS (pH 7.2) with $2 \mathrm{mM}$ EDTA and $1 \mu \mathrm{g} / \mathrm{ml}$ DAPI (BioLegend) for cell sorting. Samples were analyzed using an LSR II special-order research product (SORP) analyzer (BD Biosciences) and sorted using a FACSAria II SORP cell sorter (BD Biosciences). Data were collected and analyzed using FACSDiva 8.0.1 software (BD Biosciences).

Endothelial and stromal cell quantification. Young (3 months old) and aged (24 months old) C57BL/6 mice were intravitally labeled for 10 minutes with $25 \mu \mathrm{g}$ Alexa Fluor 647-conjugated VECAD antibody (BV13; BioLegend) via retro-orbital injection. Mice were sacrificed and femurs crushed using a mortar and pestle, and WBM was enzymatically disassociated in Hanks' balanced salt solution (Life Technologies, Thermo Fisher Scientific) containing $20 \mathrm{mM}$ HEPES (CellGro), $2.5 \mathrm{mg} / \mathrm{ml}$ collagenase A (Roche), and $1 \mathrm{U} / \mathrm{ml}$ dispase II (Roche) for 15 minutes at $37^{\circ} \mathrm{C}$ with gentle agitation. Cell suspensions were filtered $(40-\mu \mathrm{m})$, washed in PBS (pH 7.2) containing 0.5\% BSA (fraction V) and 2 mM EDTA, and depleted of terminally differentiated hematopoietic cells using a murine hematopoietic Lineage Cell Depletion Kit (Miltenyi Biotec) according to the manufacturer's instructions. To determine endothelial and stromal 
cell frequencies via flow cytometry, the resulting cell suspensions were stained using antibodies against CD31 (390; BioLegend), CD45 (30-F11; BioLegend), and TER119 (TER119; BioLegend); endothelial and stromal cell populations were defined as VECAD ${ }^{+} \mathrm{CD} 31^{+}$lineage-CD45-TER119 and VECAD ${ }^{-} \mathrm{CD} 31^{-}$lineage ${ }^{-} \mathrm{CD} 45^{-} \mathrm{TER}^{-} 19^{-}$, respectively.

Vascular permeability. To measure BM vascular integrity, Evans blue dye (Sigma-Aldrich) $0.5 \% \mathrm{w} / \mathrm{v}$ in PBS (pH 7.2) was infused via tail vein injection into young ( 3 months old) and aged (19 months old) C57BL/6 mice at $25 \mathrm{mg}$ dye $/ \mathrm{kg}$ total body weight. Three hours after injection, mice were sacrificed via cervical dislocation and cardiac perfused with $10 \mathrm{ml} \mathrm{PBS}$ (pH 7.2). Femurs were removed, denuded of excess tissue, and weighed. To extract Evans blue dye, the resulting femurs were crushed using a mortar and pestle in $600 \mu \mathrm{l}$ formamide and incubated at $55^{\circ} \mathrm{C}$ overnight. Femurs from noninjected mice were used as baseline controls. Following extraction, samples were briefly vortexed and centrifuged at $16,000 \mathrm{~g}$ for 5 minutes at room temperature. Supernatant absorbance (Abs) was recorded at $620 \mathrm{~nm}$ and $740 \mathrm{~nm}$. Sample Abs measurements were corrected for heme-containing proteins $\left[\mathrm{Abs}_{620}-\left(1.426 \times \mathrm{Abs}_{740}+\right.\right.$ 0.03)] and blanked using noninjected controls (corrected sample $\mathrm{Abs}_{620}$ - corrected noninjected control $\mathrm{Abs}_{620}$ ). Total Evans blue dye was determined using a standard curve and normalized to femur weight.

ROS. To examine ROS, young ( 3 months old) and aged ( 24 months old) C57BL/6 mice were intravitally labeled for 10 minutes with $25 \mu \mathrm{g}$ Alexa Fluor 647-conjugated VECAD antibody (BV13; BioLegend) via retro-orbital injection. Mice were sacrificed and femurs crushed using a mortar and pestle, and WBM was enzymatically disassociated in Hanks' balanced salt solution (Life Technologies, Thermo Fisher Scientific) containing $20 \mathrm{mM}$ HEPES (CellGro), $2.5 \mathrm{mg} / \mathrm{ml}$ collagenase A (Roche), and $1 \mathrm{U} / \mathrm{ml}$ dispase II (Roche) for 15 minutes at $37^{\circ} \mathrm{C}$ with gentle agitation. Cell suspensions were filtered $(40-\mu \mathrm{m})$, washed in PBS ( $\mathrm{pH}$ 7.2) containing $0.5 \%$ BSA (fraction V) and $2 \mathrm{mM}$ EDTA, and depleted of terminally differentiated hematopoietic cells using a murine hematopoietic Lineage Cell Depletion Kit (Miltenyi Biotec) according to the manufacturer's instructions. Cell suspensions were stained using antibodies against CD31 (390; BioLegend); CD45 (30-F11; BioLegend); and TER119 (TER119; Biolegend). Stained cell suspensions were then incubated with $5 \mu \mathrm{M} \mathrm{CM}-\mathrm{H} 2 \mathrm{DCFDA}$ (Thermo Fisher Scientific) in PBS at $37^{\circ} \mathrm{C}$ for 20 minutes, washed with PBS ( $\mathrm{pH} 7.2$ ) containing $0.5 \%$ BSA (fraction V) and $2 \mathrm{mM}$ EDTA, and incubated at $37^{\circ} \mathrm{C}$ for 15 minutes. EC populations were defined as $\mathrm{VECAD}^{+} \mathrm{CD} 31^{+}$lineage-CD45-TER119ROS levels were estimated using flow cytometry.

Hypoxyprobe. To evaluate BM oxygenation status, young (3 months old) and aged (24 months old) C57BL/6 mice were i.p. injected with 120 $\mathrm{mg} / \mathrm{kg}$ of $100 \mathrm{mg} / \mathrm{ml}$ pimonidazole $\mathrm{HCl}$ (Hypoxyprobe-1; Hypoxyprobe Inc.). After 90 minutes, mice were intravitally labeled for 10 minutes with $25 \mu$ g Alexa Fluor 647-conjugated VECAD antibody (BV13; BioLegend) via retro-orbital injection. For BM EC analysis by flow cytometry, mice were sacrificed and femurs were crushed using a mortar and pestle. WBM was enzymatically disassociated in Hanks' balanced salt solution (Life Technologies, Thermo Fisher Scientific) containing 20 mM HEPES (CellGro), $2.5 \mathrm{mg} / \mathrm{ml}$ collagenase A (Roche), and $1 \mathrm{U} / \mathrm{ml}$ dispase II (Roche) for 15 to 30 minutes at $37^{\circ} \mathrm{C}$ with gentle agitation. Cell suspensions were filtered $(40-\mu \mathrm{m})$, washed in PBS (pH 7.2) containing $0.5 \%$ BSA (fraction V) and 2 mM EDTA, and depleted of terminally differentiated hematopoietic cells using a murine hematopoietic Lineage Cell Depletion Kit (Miltenyi Biotec) according to the manufacturer's instructions. The resulting cell suspensions were stained using antibodies against CD31 (390; BioLegend); CD45 (30-F11; BioLegend); and TER119 (TER119; BioLegend). Following cell-surface staining, cells were fixed and permeabilized using the BD Cytofix/Cytoperm Kit (BD Biosciences) and stained with a monoclonal antibody raised against pimonidazole adducts at a 1:100 dilution (HP-Red549; Hypoxyprobe) according to the manufacturer's suggestions. EC populations were defined as VECAD ${ }^{+}$CD31+lineage-CD45-TER119- .

For BM immunohistochemical analysis, mice were sacrificed and femurs were fixed in $4 \%$ PFA in PBS ( $\mathrm{pH} 7.2$ ) overnight at $4^{\circ} \mathrm{C}$, washed in PBS (pH 7.2), and decalcified for 72 hours in $10 \%$ EDTA in PBS (pH 7.2) at room temperature with gentle rocking. Next, femurs were washed in PBS (pH 7.2), cryopreserved in $30 \%$ sucrose for 48 hours at $4^{\circ} \mathrm{C}$, and embedded in 50\% OCT (Tissue-Tek) and 50\% sucrose. Longitudinal femur sections (12- $\mu \mathrm{m}$ thickness) were cut using a CM 3050 S Cryostat (Leica), permeabilized in blocking buffer (10\% normal donkey serum [Jackson ImmunoResearch] and 0.1\% Triton X-100 in PBS, pH 7.2) for 30 minutes at room temperature, and incubated with a monoclonal antibody raised against pimonidazole adducts at a 1:100 dilution in blocking buffer (HP-Red549; Hypoxyprobe Inc.) overnight at $4^{\circ} \mathrm{C}$. Sections were washed in blocking buffer and counterstained with 1 $\mu \mathrm{g} / \mathrm{ml}$ DAPI (BioLegend) and mounted using Prolong Gold Anti-fade Solution (Life Technologies, Thermo Fisher Scientific). Femur sections were imaged on an LSM 710 confocal microscope (Zeiss).

Akt1 lentivirus. Lentivirus (21) was generated by cotransfecting pCCL-myrAkt1 backbone $(13 \mu \mathrm{g})$ with RRE $(5 \mu \mathrm{g})$, REV $(2.5 \mu \mathrm{g})$, and VSV-G $(3 \mu \mathrm{g})$ packaging plasmids onto a $10-\mathrm{cm}$ plate of $80 \%$ confluent 293T/17 cells (ATCC) using Lipofectamine 2000 (Life Technologies, Thermo Fisher Scientific) according to the manufacturer's instructions. Forty-eight hours after transfection, supernatants were processed using Lenti-X Concentrator (ClonTech) according to the manufacturer's recommendations. The resulting myrAkt1-expressing lentivirus was resuspended in $0.5 \mathrm{ml}$ TNE buffer (50 mM Tris, pH 8.0, $1 \mathrm{mM}$ EDTA, $130 \mathrm{mM} \mathrm{NaCl}$ ) and stored at $-80^{\circ} \mathrm{C}$. Viral titers were determined using the Lenti-X p24 Rapid Titer Kit (ClonTech). All transductions were done using $10,000 \mathrm{pg}$ virus per $30,000 \mathrm{ECs} / \mathrm{cm}^{2}$.

EC isolation. Primary murine endothelium was generated from young ( 3 months old) and aged ( 24 months old) C57BL/6 mice. To establish stable EC cultures, long bones were denuded of excess tissue and crushed using a mortar and pestle, and lungs were removed and minced with a surgical scalpel. Tissues were enzymatically disassociated in Hanks' balanced salt solution (Life Technologies, Thermo Fisher Scientific) containing $20 \mathrm{mM}$ HEPES (CellGro), $2.5 \mathrm{mg} / \mathrm{ml}$ collagenase A (Roche), and $1 \mathrm{U} / \mathrm{ml}$ dispase II (Roche) for 15 minutes at $37^{\circ} \mathrm{C}$ with gentle agitation. Cell suspensions were filtered $(40-\mu \mathrm{m})$, washed in PBS $(\mathrm{pH}$ 7.2) containing $0.5 \%$ BSA (fraction V) and 2 mM EDTA, and depleted of terminally differentiated hematopoietic cells using a murine hematopoietic Lineage Cell Depletion Kit (Miltenyi Biotec) according to the manufacturer's recommendations. ECs were immunopurified from the resulting cell suspensions using Dynabeads (Life Technologies, Thermo Fisher Scientific) precaptured with a monoclonal CD31 antibody (MEC13.3; BioLegend). Briefly, $4 \times 10^{6}$ sheep anti-rat IgG Dynabeads were incubated with $10 \mu \mathrm{g}$ CD31 antibody (per isolation) in $0.5 \mathrm{ml}$ PBS (pH 7.2) containing 0.5\% BSA (fraction V) and 2 mM EDTA for 30 minutes at $4^{\circ} \mathrm{C}$. Antibody-captured beads were washed 3 times in $1 \mathrm{ml}$ PBS ( $\mathrm{pH} 7.2$ ) containing $0.5 \%$ BSA (fraction V) and $2 \mathrm{mM}$ EDTA according to the manufacturer's instructions. CD31 Dynabeads were then added to lineage-depleted cell suspensions in a final volume of $1 \mathrm{ml}$ and incubated 
for 30 minutes at $4^{\circ} \mathrm{C}$. CD $31^{+}$cells were captured and washed 5 times in 1 $\mathrm{ml} \mathrm{PBS} \mathrm{(pH} \mathrm{7.2)} \mathrm{containing} \mathrm{0.5 \%} \mathrm{BSA} \mathrm{(fraction} \mathrm{V)} \mathrm{and} 2 \mathrm{mM}$ EDTA using a DynaMag-2 magnet (Life Technologies, Thermo Fisher Scientific), plated, and grown on fibronectin-coated (Sigma-Aldrich) 12-well plates (Thermo Fisher Scientific). ECs were cultured in EC growth media composed of low-glucose DMEM (Life Technologies, Thermo Fisher Scientific) and Ham's F-12 (CellGro) (1:1 ratio), supplemented with 20\% heat-inactivated FBS, antibiotic-antimycotic (CellGro), nonessential amino acids (CellGro), $10 \mathrm{mM}$ HEPES (CellGro), $100 \mu \mathrm{g} / \mathrm{ml}$ heparin (Sigma-Aldrich), and $50 \mu \mathrm{g} / \mathrm{ml}$ EC mitogen (Biomedical Technologies, Alfa Aesar). To select for Akt1-expressing ECs, cultures were grown for 7 days in serum- and cytokine-free StemSpan Serum-Free Expansion Medium (SFEM) (STEMCELL Technologies). Cells were maintained and cultured in EC growth media in humidified incubators at $37^{\circ} \mathrm{C}$ under $5 \% \mathrm{CO}_{2}$. All EC lines were subsequently stained and sorted for $\mathrm{VECAD}^{+}$(BV13; BioLegend); CD $31^{+}$ (390; BioLegend); and CD45- (30-F11; BioLegend) cell populations to ensure purity. Phase-contrast images were obtained using an Evos XL (AMG) cell imaging system. For immunocytochemistry, young and aged ECs were stained with an antibody raised against VECAD (BV13; BioLegend), counterstained with $1 \mu \mathrm{g} / \mathrm{ml}$ DAPI (BioLegend), and imaged using an LSM 710 confocal microscope (Zeiss).

AFM. Cultured ECs from young (3 months old) and aged (24 months old) C57BL/6 mice (as described above) were grown to $80 \%$ to $90 \%$ confluency on fibronectin-coated (Sigma-Aldrich) $60-\mathrm{mm}$ dishes (Corning), fixed in 4\% PFA in PBS ( $\mathrm{pH}$ 7.2) for 15 minutes at room temperature, and washed in $\mathrm{PBS}$ ( $\mathrm{pH}$ 7.2). Force maps were generated with an MFP-3D Bio AFM (Asylum Research) using a TR400PB pyramidal silicon nitride tip $(\mathrm{k}=0.02 \mathrm{~N} / \mathrm{m}, \mathrm{h}=3)$ with a $32 \times 32$ grid (1,024 force curves) covering $90 \mu \mathrm{m}^{2}$. Force curves were taken by 250 $\mathrm{nm}$ indentation with a trigger point of $200 \mathrm{pN}$ at $600 \mathrm{~nm} / \mathrm{s}$; initial $100 \mathrm{~nm}$ measurements were fitted to the Hertz-Sneddon model (53). Images were taken using an MLCT probe (Bruker) in a $90 \mu \mathrm{m}^{2}$ area with 256 points $\times 256$ lines resolution. Igor Pro 6.34A software (Asylum Research) was used to determine the cantilever spring constant via thermal tune calibration. Median endothelial stiffness is presented as a box plot. Relative stiffness was calculated by normalizing young and aged ECs to young EC stiffness measurements.

Proliferation. To assess the proliferation status of young (3 months old) and aged (24 months old) ECs in culture, fluorescence-labeled EdU incorporation was quantified by flow cytometry using a Click-iT Plus EdU Flow Cytometry Assay Kit (Thermo Fisher Scientific) according to the manufacturer's recommendations. In short, subconfluent ECs were incubated in serum-free StemSpan SFEM (STEMCELL Technologies) overnight at $37^{\circ} \mathrm{C} 5 \% \mathrm{CO}_{2}$ to synchronize the cell cycle in $\mathrm{G}_{0} /$ $\mathrm{G}_{1}$. The following day, ECs were pulsed in $10 \mu \mathrm{M}$ EdU (Alexa Fluor 488) in serum-containing growth media and analyzed at 6 and 24 hours.

Senescence. To quantify senescent cells, subconfluent young (3 months old) and aged ( 24 months old) EC cultures were analyzed by flow cytometry using the Fluorometric Quantitative Cellular Senescence Assay Kit (Cell BioLabs) according to the manufacturer's suggestions.

Wound healing. Cultured ECs from young (3 months old) and aged (24 months old) mice were plated at $10^{4}$ cells/well in a fibronectincoated (Sigma-Aldrich) 96-well ImageLock tissue culture plate (Essen Bioscience) ( $n=8$ replicate wells). At approximately $90 \%$ cell confluency, a homogenous scratch of approximately $700 \mu \mathrm{m}$ was generated using a 96-well WoundMaker (Essen Bioscience) according to the manufacturer's instructions. Wells were subsequently washed 2 times using EC growth media to remove any dislodged cells. Wound healing was monitored for 48 hours using IncuCyte ZOOM (Essen Bioscience) and calculated as a percentage of the original wound mask at 12-hour intervals. Relative wound density (RWD) was calculated using the following equation: percentage of $\mathrm{RWD}_{(t)}=100 \times\left(\mathrm{w}_{(t)}-\mathrm{w}_{(0)}\right) /\left(\mathrm{c}_{(t)}-\mathrm{w}_{(0)}\right)$, where $\mathrm{w}_{(t)}=$ density of the wound region at the time point $(t)$ and $\mathrm{c}_{(t)}=$ density of the cell region at the time point $(t)$.

Gene expression. For gene expression analysis, total RNA from young (3 months old) and aged (24 months old) cultured endothelium was isolated using TRIzol Reagent (Thermo Fisher Scientific), and cDNA was generated from $2 \mu \mathrm{g}$ total RNA using SuperScript III Reverse Transcriptase (Thermo Fisher Scientific) according to the manufacturer's suggested protocol. RT-qPCR analysis was performed using a 40-ng cDNA template with $1 \mu \mathrm{M}$ gene-specific primers and $1 \times$ SYBR Green Master Mix (Applied Biosystems) on a ViiA7 Real-time PCR System (Applied Biosystems). The appropriate minus-reverse transcriptase controls were included in all experiments. Primer sets were obtained from the Harvard Primer Bank or designed in-house (54-56). For a complete list of the primers used, see Supplemental Table 1.

Ex vivo coculture. Primary murine young (3 months old) or aged ( 24 months old) HSPCs were cocultured on young or aged Akt-transduced endothelium in serum-free conditions with sKITL supplementation for 14 days. To establish cocultures, femurs and tibiae from young and aged C57BL/6 (CD45.2 $2^{+}$mice were isolated, and WBM was flushed using a 26.5-gauge needle with PBS ( $\mathrm{pH} 7.2$ ) containing 0.5\% BSA (fraction V) and $2 \mathrm{mM}$ EDTA. Isolated WBM was subsequently depleted of hematopoietic lineage-committed cells using a murine hematopoietic Lineage Cell Depletion Kit (Miltenyi Biotec) according to the manufacturer's instructions. Lineage-depleted HSPCs $\left(10^{5} /\right.$ well) were plated on a single well of a 12-well dish with confluent young or aged endothelium in $1 \mathrm{ml}$ serum-free StemSpan SFEM (STEMCELL Technologies) with $50 \mathrm{ng} / \mathrm{ml}$ recombinant murine sKITL (PeproTech). Three independently isolated HSPC populations were tested in parallel for each EC line. Cocultures were incubated at $37^{\circ} \mathrm{C}$ and $5 \% \mathrm{CO}_{2}$ for a total of 14 days. Day 2: Cocultures were supplemented with $1 \mathrm{ml}$ StemSpan SFEM and $50 \mathrm{ng} / \mathrm{ml}$ sKITL. Day 4: Nonadherent hematopoietic cells were collected, pelleted $(500 \times g$ for $5 \mathrm{~min})$, resuspended in $3 \mathrm{ml}$ StemSpan SFEM, and split 1:2 $(1.5 \mathrm{ml} /$ well $)$ into the original and adjacent 12 wells with ECs $(2 \times 12$ wells total). Cocultures were supplemented with $50 \mathrm{ng} / \mathrm{ml}$ sKITL. Day 6: Nonadherent hematopoietic cells were collected and split 1:2 into 12 adjacent wells with StemSpan SFEM to a final volume of $1.5 \mathrm{ml}(4 \times 12$ wells total $)$. Cocultures were supplemented with $50 \mathrm{ng} / \mathrm{ml}$ sKITL. Day 7: Nonadherent hematopoietic cells were collected and depleted of lineage-committed cells using a murine hematopoietic Lineage Cell Depletion Kit. Lineage-depleted HSPCs $\left(10^{5} /\right.$ well $)$ were plated in a single well of a new 12-well plate with confluent young or aged endothelium in $1 \mathrm{ml}$ StemSpan SFEM with $50 \mathrm{ng} / \mathrm{ml}$ sKITL. StemSpan SFEM (1 ml/well) was added back to the original 12-well plate with $50 \mathrm{ng} / \mathrm{ml}$ sKITL. Days 8-13: A new 12-well plate was split as described above. StemSpan SFEM was exchanged every 2 days and replated with $50 \mathrm{ng} / \mathrm{ml}$ sKITL on the original 12-well coculture plate. Day 14: Endothelial and hematopoietic cells were collected for analysis using Accutase (BioLegend). Cell numbers were determined using hemocytometer counts with trypan blue (Life Technologies, Thermo Fisher Scientific) for live/dead exclusion. Following coculture, total hematopoietic cell and HSPC frequencies were assessed using antibodies against CD45 
(30-F11; BioLegend); GR1 (RB6-8C5; BioLegend); CD11B (M1/70; BioLegend), B220 (RA3-6B2; BioLegend); CD3 (17A2; BioLegend); CD41 (MWReg30; BioLegend); cKIT (2B8; BioLegend); and SCA1 (D7; BioLegend). To assess HSC-repopulating activity, cocultures were enriched for hematopoietic cells using murine CD45 Microbeads (Miltenyi Biotec); $5 \times 10^{5} \mathrm{CD} 45.2^{+}$(C57BL/6) cocultured cells were transplanted into lethally irradiated $(9.50 \mathrm{~Gy})$ young $(3$ months old) CD45.1 $1^{+}$(B6.SJL-Ptprc ${ }^{a} P e p c^{b} /$ BoyJ) recipients with $5 \times 10^{5}$ young (3 months old) CD $45.1^{+}$competitive WBM cells (per mouse) via retroorbital sinus injection. Multilineage engraftment in PB was assayed 4 months after transplantation using antibodies raised against GR1 (RB6-8C5; BioLegend); CD11B (M1/70; BioLegend); B220 (RA36B2; BioLegend); CD19 (1D3; BD Biosciences); CD4 (GK1.5; BioLegend); and CD8 at 1:100 (53-6.7; BioLegend). The antibodies used for hematopoietic engraftment were CD45.1 (A20; BioLegend); CD45.2 (104; BioLegend); and TER119 (TER119; BioLegend).

$P B$ analysis. $\mathrm{PB}$ was collected using 75-mm heparinized glass capillary tubes (Kimble-Chase) via retro-orbital sinus bleeds. To assess hematopoietic recovery following myeloablation, $\mathrm{CBCs}$ were analyzed using an ADVIA 120 Hematology System (Bayer Healthcare). To quantify multilineage hematopoietic and HSC engraftment, PB was depleted of RBC (BioLegend), stained with preconjugated antibodies, and analyzed by flow cytometry.

EC infusions. Young (3 months old) and aged (24 months old) C57BL/6 (CD45.2) mice were subjected to a myelosuppressive dose of TBI (6.50 Gy) and infused with $5 \times 10^{5}$ young ( 3 months old) or $5 \times 10^{5}$ aged (24 months old) ECs via retro-orbital sinus injections on 4 successive days. PBS (vehicle) injections and steady-state mice (nonirradiated) were used as controls. PB CBCs were analyzed for hematopoietic recovery weekly for 28 days following TBI. Preirradiated animals were bled to establish CBC baselines. To assess HSC activity following EC infusion, femurs were isolated 28 days after TBI and WBM was flushed using a 26.5-gauge needle with PBS (pH 7.2) containing 0.5\% BSA (fraction V) and 2 mM EDTA. Viable WBM cell counts were determined using a hemocytometer and trypan blue (Life Technologies, Thermo Fisher Scientific). $2 \times 10^{6} \mathrm{CD} 45.2^{+}$donor WBM cells were transplanted with $10^{6}$ competitive CD 45.1 ${ }^{+}$WBM cells into lethally-irradiated (9.5 Gy) young (3 months old) CD45.1 ${ }^{+}$(B6.SJL-PtprcaPepcb/BoyJ) recipients via retroorbital sinus injections. Multilineage engraftment in PB was assayed 4 months after transplantation using antibodies raised against GR1 (RB68C5; BioLegend); CD11B (M1/70; BioLegend); B220 (RA3-6B2; BioLegend); and CD3 (17A2; BioLegend). Antibodies raised against CD45.1 (A20; BioLegend); CD45.2 (104; BioLegend); and TER119 (TER119; BioLegend) were used for hematopoietic engraftment.

Coinfusions of ECs and WBM cells. Femurs and tibiae were harvested from young ( 3 months old) and aged ( 24 months old) C57BL/6 mice and flushed using a 26.5-gauge needle with PBS (pH 7.2) containing $0.5 \%$ BSA (fraction V) and 2 mM EDTA and then counted using hemocytometers and trypan blue (Life Technologies, Thermo Fisher Scientific) for live/dead exclusion. WBM cells $\left(10^{5}\right.$ cells) were transplanted into lethally irradiated ( $9.50 \mathrm{~Gy}$ ) young ( 3 months old) C57BL/6 recipients, with or without $5 \times 10^{5}$ young ECs, via retro-orbital injection. PBS (vehicle) and steady-state mice (nonirradiated) were used as controls. Hematopoietic recovery was monitored by PB CBCs. Mice were monitored daily to generate survival curves. To assess CFU-S, spleens were removed 8 days after TBI, fixed in Bouin's solution (Sigma-Aldrich) overnight, and macroscopic surface colonies quantified (48). Tissue histology was performed by Histoserv Inc. Briefly, tissues were fixed in $4 \%$ PFA in PBS ( $\mathrm{pH} 7.2$ ) overnight at $4^{\circ} \mathrm{C}$, washed in PBS ( $\mathrm{pH} 7.2$ ), and subsequently dehydrated in $70 \%$ ethanol. Femurs were decalcified for 72 hours in 10\% EDTA in PBS (pH 7.2) at room temperature with gentle rocking prior to dehydration. Paraffin-embedded femur, splenic, and small intestine tissues were sectioned (6- $\mu \mathrm{m}$ thickness) and stained with H\&E (Histoserv Inc). Paraffin-embedded femurs were processed for VEGFR $3^{+}$sinusoidal vessel regression analysis using an antibody raised against VEGFR3 (AFL4; BioLegend) and counterstained with hematoxylin as previously described (43). Images were taken on a BX51 (Olympus) light microscope at $\times 10$ or $\times 20$ magnification. To quantify BM hematopoietic recovery, femurs were crushed using a mortar and pestle in PBS ( $\mathrm{pH}$ 7.2) containing $0.5 \%$ BSA (fraction V) and $2 \mathrm{mM}$ EDTA and stained using antibodies raised against CD45 (30-F11; BioLegend); GR1 (RB6-8C5; BioLegend); CD115 (AFS98; BioLegend); and F4/80 (BM8; BioLegend).

Limiting dilution. Femurs and tibiae were harvested from young (3 months old) and aged (24 months old) C57BL/6 mice and flushed using a 26.5-gauge needle with PBS ( $\mathrm{pH} 7.2$ ) containing 0.5\% BSA (fraction V) and $2 \mathrm{mM}$ EDTA, and then cells were counted using a hemocytometer and trypan blue (Life Technologies, Thermo Fisher Scientific) for live/ dead exclusion. For limiting dilution analysis, the indicated numbers (Figure 5, G and H, and Supplemental Figure 4, B and C) of WBM cells were transplanted into lethally irradiated young ( 3 months old) C57BL/6 recipients (9.50 Gy) in a noncompetitive manner (per mouse) via retroorbital injection ( $n=10 \mathrm{mice} / \mathrm{cohort}$ ). The percentage of negativeresponding/dead mice was monitored throughout a 4-month post-transplantation period. Surviving mice were analyzed for multilineage engraftment in the PB using antibodies raised against GR1 (RB6-8C5; BioLegend); CD11B (M1/70; BioLegend); B220 (RA3-6B2; BioLegend); and CD3 (17A2; BioLegend). Long-term multilineage HSC frequency and $95 \%$ CIs were determined using extreme limiting dilution analysis (ELDA) software (http://bioinf.wehi.edu.au/software/elda/) (57).

Hematopoietic homing analysis. Femurs and tibiae were harvested from young (3 months old) and aged (24 months old) C57BL/6 (CD45.2 $)$ mice and flushed using a 26.5-gauge needle with PBS (pH 7.2) containing $0.5 \%$ BSA (fraction V) and $2 \mathrm{mM}$ EDTA, and then counted using a hemocytometer and trypan blue (Life Technologies, Thermo Fisher Scientific) for live/dead exclusion. For hematopoietic homing assessment, $10^{6}$ young or aged WBM cells, with or without $5 \times 10^{5}$ young ( 3 months old) ECs, were infused or coinfused into young ( 3 months old) lethally irradiated (9.50 Gy) CD45.1 (B6.SJL-Ptprc ${ }^{a} P e p c^{b} /$ BoyJ) recipients. Femurs and spleens were removed 16 hours after infusion, flushed with PBS (pH 7.2) containing 0.5\% BSA (fraction V) and 2 mM EDTA, and lysed for RBC (BioLegend). The resulting cells were then stained for antibodies raised against CD45.1 (A20; BioLegend); CD45.2 (104; BioLegend); and TER119 (TER119; BioLegend). CD45.2 ${ }^{+}$hematopoietic cells were quantified by flow cytometry as a frequency per total WBM or splenocytes.

Statistics. No statistical models were used to predetermine experimental sample sizes. Unless otherwise noted, all bar graphs and line graphs are displayed as the mean \pm SEM, and significance was determined using an unpaired, 2-tailed Student's $t$ test, with the threshold set at a $P$ value of less than 0.05. Median endothelial stiffness and significance (Figure 2D) were determined using a nonparametric, 1-sided Wilcoxon rank-sum test ( $\alpha=0.001$ ) to compare repeated force measurements. Data are presented as box plots, with whiskers representing an interquartile range (IQR) of \pm 1.5 . Relative endothelial stiffness (Figure $2 \mathrm{E}$ ) was nor- 
malized to young ECs and presented as a 95\% CI. Survival curves (Figure 5 , A and B) were calculated using the log-rank test to compare WBM with WBM-plus-EC cohorts. Long-term multilineage HSC frequency and 95\% CIs (Figure 5, G and $\mathrm{H}$ ) were determined using ELDA software (57).

Study approval. All mouse experiments were performed with approval of the IACUC of Weill Cornell Medical College and in accordance with the guidelines of the Association for Assessment and Accreditation of Laboratory Animal Care - International (AAALAC) and the NIH Office of Laboratory Animal Welfare (OLAW).

\section{Author contributions}

MGP, PR, MCG, and JMB performed experiments and conducted data analyses. PL, KG, and SYR performed and analyzed AFM experiments. MGP and JMB conceived the experiments and wrote the manuscript.

\section{Acknowledgments}

We would like to thank the Starr Foundation Tri-Institutional core facilities. Statistical consultation was provided by Paul Christos and supported by the Clinical and Translational Science Center (UL1-TR000457-06) in the Division of Biostatistics, Department of Healthcare Policy and Research of Weill Cornell Medical College. Our work is supported by the Tri-Institutional Stem Cell Initiative (2014-004); an American Society of Hematology Scholar Award; the American Federation for Aging Research; the NIH (1R01CA204308 and 1R01HL133021); and the Leukemia and Lymphoma Society Quest for Cures.

Address correspondence to: Jason M. Butler, Weill Cornell Medical College, 1300 York Avenue, A-827A, New York, New York 10065, USA. Phone: 212.746.2673; Email:jmb2009@med.cornell.edu.
1. Federal Interagency Forum On Aging Related Statistics. Older Americans 2016: Key Indicators of Well-Being. Aging Stats Website. https://agingstats.gov/data.html. Accessed September 7, 2017.

2. Schlitzkus LL, Melin AA, Johanning JM, Schenarts PJ. Perioperative management of elderly patients. Surg Clin North Am. 2015;95(2):391-415.

3. North BJ, Sinclair DA. The intersection between aging and cardiovascular disease. Circ Res. 2012;110(8):1097-1108.

4. Berger NA, et al. Cancer in the elderly. Trans Am Clin Climatol Assoc. 2006;117:147-155; discussion 155.

5. Dorshkind K, Swain S. Age-associated declines in immune system development and function: causes, consequences, and reversal. Curr Opin Immunol. 2009;21(4):404-407.

6. Eisenstaedt R, Penninx BW, Woodman RC. Anemia in the elderly: current understanding and emerging concepts. Blood Rev. 2006;20(4):213-226.

7. Lichtman MA, Rowe JM. The relationship of patient age to the pathobiology of the clonal myeloid diseases. Semin Oncol. 2004;31(2):185-197.

8. Pang WW, Schrier SL, Weissman IL. Age-associated changes in human hematopoietic stem cells. Semin Hematol. 2017;54(1):39-42.

9. Cho RH, Sieburg HB, Muller-Sieburg CE. A new mechanism for the aging of hematopoietic stem cells: aging changes the clonal composition of the stem cell compartment but not individual stem cells. Blood. 2008;111(12):5553-5561.

10. Dykstra B, de Haan G. Hematopoietic stem cell aging and self-renewal. Cell Tissue Res. 2008;331(1):91-101.

11. Rossi DJ, et al. Cell intrinsic alterations underlie hematopoietic stem cell aging. Proc Natl Acad Sci U S A. 2005;102(26):9194-9199.

12. Van Zant G, Liang Y. The role of stem cells in aging. Exp Hematol. 2003;31(8):659-672.

13. Pang WW, et al. Human bone marrow hematopoietic stem cells are increased in frequency and myeloid-biased with age. Proc Natl Acad Sci US A. 2011;108(50):20012-20017.

14. Chambers SM, Shaw CA, Gatza C, Fisk CJ, Donehower LA, Goodell MA. Aging hematopoietic stem cells decline in function and exhibit epigenetic dysregulation. PLoS Biol. 2007;5(8):e201.

15. Geiger H, de Haan G, Florian MC. The ageing haematopoietic stem cell compartment. Nat Rev
Immunol. 2013;13(5):376-389.

16. Elias HK, Bryder D, Park CY. Molecular mechanisms underlying lineage bias in aging hematopoiesis. Semin Hematol. 2017;54(1):4-11.

17. Akunuru S, Geiger H. Aging, Clonality, and Rejuvenation of Hematopoietic Stem Cells. Trends Mol Med. 2016;22(8):701-712.

18. Morrison SJ, Scadden DT. The bone marrow niche for haematopoietic stem cells. Nature. 2014;505(7483):327-334.

19. Ding L, Saunders TL, Enikolopov G, Morrison SJ. Endothelial and perivascular cells maintain haematopoietic stem cells. Nature. 2012;481(7382):457-462.

20. Ding L, Morrison SJ. Haematopoietic stem cells and early lymphoid progenitors occupy distinct bone marrow niches. Nature. 2013;495(7440):231-235.

21. Kobayashi H, et al. Angiocrine factors from Akt-activated endothelial cells balance selfrenewal and differentiation of haematopoietic stem cells. Nat Cell Biol. 2010;12(11):1046-1056.

22. Butler JM, et al. Endothelial cells are essential for the self-renewal and repopulation of Notch-dependent hematopoietic stem cells. Cell Stem Cell. 2010;6(3):251-264.

23. Poulos MG, et al. Endothelial Jagged-1 is necessary for homeostatic and regenerative hematopoiesis. Cell Rep. 2013;4(5):1022-1034.

24. Greenbaum A, et al. CXCL12 in early mesenchymal progenitors is required for haematopoietic stem-cell maintenance. Nature. 2013;495(7440):227-230.

25. Himburg HA, et al. Pleiotrophin regulates the expansion and regeneration of hematopoietic stem cells. Nat Med. 2010;16(4):475-482.

26. Donnini A, Re F, Orlando F, Provinciali M. Intrinsic and microenvironmental defects are involved in the age-related changes of $\mathrm{Lin}^{-} \mathrm{c}-\mathrm{kit}^{+}$ hematopoietic progenitor cells. Rejuvenation Res. 2007;10(4):459-472.

27. Kusumbe AP, et al. Age-dependent modulation of vascular niches for haematopoietic stem cells. Nature. 2016;532(7599):380-384.

28. Vas V, Senger K, Dörr K, Niebel A, Geiger $\mathrm{H}$. Aging of the microenvironment influences clonality in hematopoiesis. PLoS One. 2012;7(8):e42080.
29. Vas V, Wandhoff C, Dörr K, Niebel A, Geiger H. Contribution of an aged microenvironment to aging-associated myeloproliferative disease. PLoS One. 2012;7(2):e31523.

30. Guidi N, et al. Osteopontin attenuates agingassociated phenotypes of hematopoietic stem cells. EMBO J. 2017;36(7):840-853.

31. Claesson-Welsh L. Vascular permeability - the essentials. Ups J Med Sci. 2015;120(3):135-143.

32. Pober JS, Sessa WC. Evolving functions of endothelial cells in inflammation. Nat Rev Immunol. 2007;7(10):803-815.

33. Csiszar A, Wang M, Lakatta EG, Ungvari Z. Inflammation and endothelial dysfunction during aging: role of NF-кB. J Appl Physiol. 2008;105(4):1333-1341.

34. El Assar M, Angulo J, Rodríguez-Mañas L. Oxidative stress and vascular inflammation in aging. Free Radic Biol Med. 2013;65:380-401.

35. Mittal M, Siddiqui MR, Tran K, Reddy SP, Malik AB. Reactive oxygen species in inflammation and tissue injury. Antioxid Redox Signal. 2014;20(7):1126-1167.

36. Zhang C. The role of inflammatory cytokines in endothelial dysfunction. Basic Res Cardiol. 2008;103(5):398-406.

37. Poulos MG, et al. Vascular platform to define hematopoietic stem cell factors and enhance regenerative hematopoiesis. Stem Cell Reports. 2015;5(5):881-894.

38. Paneni F, Diaz Cañestro C, Libby P, Lüscher TF, Camici GG. The aging cardiovascular system: understanding it at the cellular and clinical levels. J Am Coll Cardiol. 2017;69(15):1952-1967.

39. Tian XL, Li Y. Endothelial cell senescence and age-related vascular diseases. J Genet Genomics. 2014;41(9):485-495.

40. Brandes RP, Fleming I, Busse R. Endothelial aging. Cardiovasc Res. 2005;66(2):286-294.

41. Tuljapurkar SR, et al. Changes in human bone marrow fat content associated with changes in hematopoietic stem cell numbers and cytokine levels with aging. J Anat. 2011;219(5):574-581.

42. Latchney SE, Calvi LM. The aging hematopoietic stem cell niche: Phenotypic and functional changes and mechanisms that contribute to hematopoietic aging. Semin Hematol. 2017;54(1):25-32. 
43. Poulos MG, et al. Endothelial-specific inhibition of NF- $\mathrm{BB}$ enhances functional haematopoiesis. Nat Commun. 2016;7:13829.

44. Chute JP, et al. Transplantation of vascular endothelial cells mediates the hematopoietic recovery and survival of lethally irradiated mice. Blood. 2007;109(6):2365-2372.

45. Salter AB, et al. Endothelial progenitor cell infusion induces hematopoietic stem cell reconstitution in vivo. Blood. 2009;113(9):2104-2107.

46. Montfort MJ, Olivares CR, Mulcahy JM, Fleming WH. Adult blood vessels restore host hematopoiesis following lethal irradiation. Exp Hematol. 2002;30(8):950-956.

47. Balducci L. Myelosuppression and its consequences in elderly patients with cancer. Oncology (Williston Park, NY). 2003;17(11 suppl 11):27-32.

48. Till JE, McCulloch EA. A direct measurement of the radiation sensitivity of normal mouse bone marrow cells. Radiat Res. 1961;14:213-222.
49. Li XM, Hu Z, Jorgenson ML, Wingard JR, Slayton WB. Bone marrow sinusoidal endothelial cells undergo nonapoptotic cell death and are replaced by proliferating sinusoidal cells in situ to maintain the vascular niche following lethal irradiation. Exp Hematol. 2008;36(9):1143-1156.

50 . Hooper AT, et al. Engraftment and reconstitution of hematopoiesis is dependent on VEGFR2mediated regeneration of sinusoidal endothelial cells. Cell Stem Cell. 2009;4(3):263-274.

51. Ergen AV, Boles NC, Goodell MA. Rantes/ Ccl5 influences hematopoietic stem cell subtypes and causes myeloid skewing. Blood. 2012;119(11):2500-2509.

52. Li B, Bailey AS, Jiang S, Liu B, Goldman DC, Fleming WH. Endothelial cells mediate the regeneration of hematopoietic stem cells. Stem Cell Res. 2010;4(1):17-24.

53. Wong AK, Llanos P, Boroda N, Rosenberg SR, Rabbany SY. A parallel-plate flow chamber for mechanical characterization of endothelial cells exposed to laminar shear stress. Cell Mol Bioeng. 2016;9(1):127-138.

54. Spandidos A, Wang X, Wang H, Dragnev S, Thurber T, Seed B. A comprehensive collection of experimentally validated primers for Polymerase Chain Reaction quantitation of murine transcript abundance. BMC Genomics. 2008;9:633.

55. Spandidos A, Wang X, Wang H, Seed B. PrimerBank: a resource of human and mouse PCR primer pairs for gene expression detection and quantification. Nucleic Acids Res. 2010;38(Database issue):D792-D799.

56. Wang X, Seed B. A PCR primer bank for quantitative gene expression analysis. Nucleic Acids Res. 2003;31(24):e154.

57. Hu Y, Smyth GK. ELDA: extreme limiting dilution analysis for comparing depleted and enriched populations in stem cell and other assays. J Immunol Methods. 2009;347(1-2):70-78. 